TITLE:

\title{
Vacuum formation behind the expansion wave in a piston motion problem
}

\section{$\operatorname{AUTHOR(S):~}$}

Taguchi, Satoshi; Takata, Shigeru

\section{CITATION:}

Taguchi, Satoshi ...[et al]. Vacuum formation behind the expansion wave in a piston motion problem. Physical Review E 2012, 86(1): 016305.

ISSUE DATE:

2012-07-06

URL:

http://hdl.handle.net/2433/188011

RIGHT:

C)2012 American Physical Society 
PHYSICAL REVIEW E 86, 016305 (2012)

\title{
Vacuum formation behind the expansion wave in a piston motion problem
}

\author{
Satoshi Taguchi \\ Department of Mechanical Engineering and Intelligent Systems, University of Electro-Communications, Chofu, Tokyo 182-8585, Japan \\ Shigeru Takata* \\ Department of Mechanical Engineering and Science and Advanced Research Institute of Fluid Science and Engineering, \\ Kyoto University, Kyoto 606-8501, Japan \\ (Received 21 March 2012; published 6 July 2012)
}

\begin{abstract}
Long time behavior of one-dimensional gas motion caused by pulling a piston with a high speed is numerically studied on the basis of the kinetic theory of gases. It is clarified that (i) if the piston speed is lower than a critical speed, the state of the gas time-asymptotically approaches the local equilibrium that corresponds to the isentropic solution in the conventional gas dynamics, namely, an expansion wave followed by a uniform state, and (ii) otherwise, there appears a highly nonequilibrium region with a very low pressure behind the expansion tail. In the latter case, the components of temperature parallel and perpendicular to the flow direction become highly different, and the density tends to vanish as time goes on, forming a vacuum region behind the tail at the infinite time.

DOI: 10.1103/PhysRevE.86.016305

PACS number(s): 47.40.Ki, 47.45.-n, 51.10.+y, 47.85.Gj
\end{abstract}

\section{INTRODUCTION}

Formation of a one-dimensional shock or expansion wave and its propagation in a gas caused by a piston motion is one of the classical and fundamental problems in gas dynamics. The behavior of the gas is well described by the theory of compressible perfect fluid, namely, by the compressible Euler set of equations [1].

In the case of pulling out a piston, the expansion wave is generated and propagates in the direction opposite to the piston velocity, relative to the piston. The expansion front propagates with a sound speed relative to the upstream, followed by a region with a uniform low pressure and temperature. The faster the piston moves, the lower the downstream pressure is, and the downstream uniform pressure becomes zero when the piston speed exceeds a critical speed. This is the formation of vacuum in the framework of the conventional gas dynamics (CGD). We are mainly interested in this process of vacuum formation.

The above physical picture is based on the conventional gas dynamics, namely, the compressible Euler set of equations, where the local equilibrium state of a gas is assumed as a consequence of frequent collisions among gas molecules. This assumption is, however, no longer valid if the downstream pressure becomes extremely low. We revisit the above problem from the viewpoint of the kinetic theory of gases.

The present work is motivated by Ref. [2], which treats strong evaporating flows from a plane condensed phase in the case of a binary mixture. In this reference, the second author studies the long time behavior of the mixture and concludes a possibility of two traveling expansion waves separated by a vacuum region. In the literature, there are also several works [3-6] based on the kinetic theory that aim at the physical investigation of the frozen temperature in steady two- and three-dimensional near vacuum flows. The near vacuum gas is also a fundamental mathematical subject in the kinetic theory (e.g., Refs. [7-10]); the existence of a global solution [7,8]

*takata.shigeru.4a@kyoto-u.ac.jp. of the Boltzmann equation in an infinite domain and its stability [9] have been reported. However, little is known on the formation of vacuum behind the expansion tail on the basis of the kinetic theory. We have only found some works on a stable numerical scheme for solving the compressible Euler set of equations in low pressure regions, on the scheme for capturing a sharp front of a gas into a real vacuum [11,12], on the gas-dynamic stability of one-dimensional free expansion into vacuum [13], and on a collisionless flow of this free expansion [14].

In a near vacuum, the intermolecular collisions rarely occur. As we show later, the feature of the free molecular flow (the collisionless gas limit) is remarkably different from that of the isentropic flow predicted by the conventional gas dynamics. We will numerically clarify the behavior of the gas flow that contains both the region of the expansion wave where the intermolecular collisions are dominant and the highly low pressure region behind the expansion tail where a transition to the free-molecular-flow-like feature is expected.

\section{PROBLEM}

Keeping in mind the physical situation of the piston motion problem, we consider a somewhat artificial problem described below. In the last paragraph of the section, we explain the points that make our formulation a little artificial.

Consider a monatomic perfect gas bounded by a resting boundary A located at $X_{1}=0$ and by a moving adiabatic boundary B, where $X_{i}$ is the Cartesian coordinate system. The boundary $\mathrm{B}$ is located at $X_{1}=0$ at the initial time $t=0$ and moves in the $X_{1}$ direction with a positive constant velocity $u_{p}>0$ (Fig. 1). Molecules approaching the boundary A are absorbed on its surface, while molecules are emitted from the surface according to the half Maxwellian distribution characterized by mass density $\rho_{0}$, temperature $T_{0}$, and flow velocity $\left(a_{0}, 0,0\right)$. Here, $a_{0}=\sqrt{\gamma R T_{0}}$ is the sound speed at temperature $T_{0}, \gamma=5 / 3$ is the specific heat ratio, and $R$ is the 


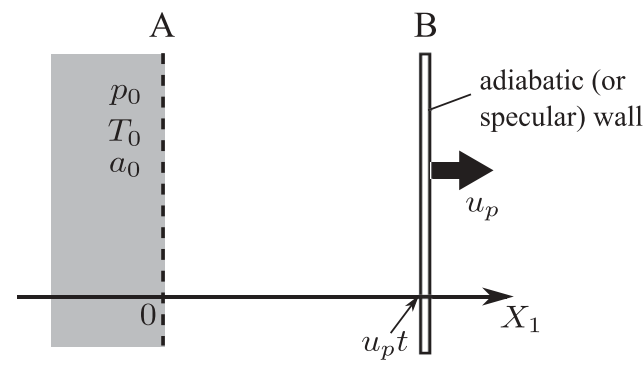

FIG. 1. Sketch of problem: the gas behavior induced by pulling the adiabatic wall of piston $\mathrm{B}$ with velocity $u_{p}$. A is an artificial boundary that models the undisturbed sonic state with pressure $p_{0}$, temperature $T_{0}$, and flow velocity $a_{0}$ ( $a_{0}$ : the sound speed). The expansion wave will be formed with its front always at $X_{1}=0$ because the upstream is the sonic state.

specific gas constant. $u_{p}>a_{0}$ is assumed. On the boundary B, molecules are specularly reflected.

We assume that the gas behavior is described by the Bhatnagar-Gross-Krook (BGK) (or Boltzmann-KrookWelander) model of the Boltzmann equation $[15,16]$. Then, denoting the molecular velocity by $\xi$ and the velocity distribution function by $f\left(t, X_{1}, \xi\right)$, the problem is written by the following equation

$$
\begin{aligned}
\frac{\partial f}{\partial t}+\xi_{1} \frac{\partial f}{\partial X_{1}} & =A_{c} \rho(M-f),\left(0<X_{1}<u_{p} t\right), \\
M & =\frac{\rho}{(2 \pi R T)^{3 / 2}} \exp \left[-\frac{\left(\xi_{1}-u\right)^{2}+\xi_{2}^{2}+\xi_{3}^{2}}{2 R T}\right], \\
\rho & =\int f d \xi, \quad \rho u=\int \xi_{1} f d \xi \\
p & =\rho R T=\frac{1}{3} \int\left[\left(\xi_{1}-u\right)^{2}+\xi_{2}^{2}+\xi_{3}^{2}\right] f d \xi
\end{aligned}
$$

the boundary conditions

$$
\begin{aligned}
f & =M_{A},\left(X_{1}=0, \xi_{1}>0\right), \\
f\left(\xi_{1}\right) & =f\left(\xi_{1}-2\left(\xi_{1}-u_{p}\right)\right),\left(X_{1}=u_{p} t, \xi_{1}<u_{p}\right),
\end{aligned}
$$

(a)

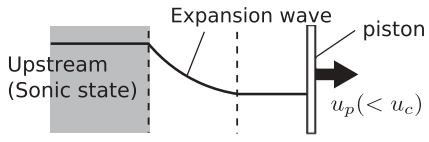

(b)

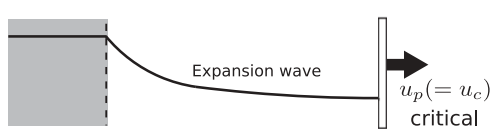

(c)

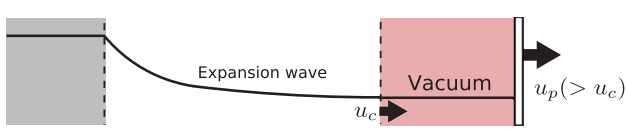

FIG. 2. (Color online) Schematic depiction of the pressure profile in the expansion wave and its downstream predicted by the conventional gas dynamics. (a) $u_{p}<u_{c}$, (b) $u_{p}=u_{c}$, and (c) $u_{p}>u_{c}$. Here, $u_{p}$ is the piston speed, while $u_{c}=\frac{\gamma+1}{\gamma-1} a_{0}\left(=4 a_{0}\right)$ is the critical speed. The vacuum region appears in case (c), where the piston speed exceeds the critical speed. and the initial condition

$$
f=\lim _{t \rightarrow 0, X_{1} \rightarrow 0} f_{0}\left(t, X_{1}, \boldsymbol{\xi}\right) .
$$

Here, $A_{c}$ is a constant ( $\rho A_{c}$ is a collision frequency); $f_{0}$ is given by

$$
\begin{aligned}
f_{0}\left(t, X_{1}, \xi\right) & = \begin{cases}M_{A}, & \left(\xi_{1}>X_{1} / t\right) \\
M_{B}, & \left(\xi_{1}<X_{1} / t\right),\end{cases} \\
M_{A} & =\frac{\rho_{0}}{\left(2 \pi R T_{0}\right)^{3 / 2}} \exp \left[-\frac{\left(\xi_{1}-a_{0}\right)^{2}+\xi_{2}^{2}+\xi_{3}^{2}}{2 R T_{0}}\right] \\
M_{B}(\xi) & =M_{A}\left(\xi_{1}-2\left(\xi_{1}-u_{p}\right), \xi_{2}, \xi_{3}\right),
\end{aligned}
$$

and satisfies Eq. (1a) in the free molecular limit $\left(A_{c}=0\right)$; and $\rho,(u, 0,0), T$, and $p$ are the mass density, flow velocity, temperature, and pressure of the gas, respectively. Here and in what follows, the arguments of functions are suppressed whenever there is no ambiguity. Note that we have assumed a one-dimensional flow from the beginning. Because the gas region is infinitely thin at the initial time, the initial condition (3) is given somewhat in an irregular way. For later convenience, we also define the components of the pressure and temperature, $\left(p_{\|}, T_{\|}\right)$and $\left(p_{\perp}, T_{\perp}\right)$, in the directions parallel and perpendicular to the flow by

$$
\begin{aligned}
& p_{\|}=\rho R T_{\|}=\int\left(\xi_{1}-u\right)^{2} f d \xi, \\
& p_{\perp}=\rho R T_{\perp}=\frac{1}{2} \int\left(\xi_{2}^{2}+\xi_{3}^{2}\right) f d \xi .
\end{aligned}
$$

Hereinafter, $p_{\|}$and $T_{\|}$will be called the parallel pressure and temperature, while $p_{\perp}$ and $T_{\perp}$ will be called the perpendicular pressure and temperature.

We make some remarks on the above formulation. We expect a generation of an expansion wave propagating toward upstream. Since the expansion front propagates with a sound speed relative to the stream, it is at rest in the present coordinate frame in which the upstream is in a uniform sonic state. The boundary A models this upstream sonic state, so that the expansion front always locates at $X_{1}=0$. The boundary B models the adiabatic piston wall; accordingly $M_{B}$ is just a mirror image of $M_{A}$ with respect to $\mathrm{B}$. In the standard formulation of the piston motion problem, the gas would occupy a half space $X_{1}<u_{p} t$, condition (2a) would be imposed at $X_{1} \rightarrow-\infty$, and the initial condition would be $f=M_{A}$ for all $X_{1}(<0)$ and $\xi$. The difference of our formulation from the standard one lies in a trigger inducing the expansion wave. In the standard formulation, the expansion wave is triggered by a sudden change of piston velocity from $a_{0}$ to $u_{p}$ when it passes $X_{1}=0$ at $t=0$. On the other hand, in our formulation, the piston velocity $u_{p}$ is unchanged for the entire time $(-\infty<t<\infty)$. Instead, the piston motion in a free molecular gas is considered for $t<0$ and the expansion wave is triggered by turning on intermolecular collisions after $t=0$ when the piston passes the origin $X_{1}=0$.

\section{TRANSFORMATION OF VARIABLES}

The problem formulated in Sec. II can be conveniently rewritten by using $X_{1} / t$ in place of $X_{1}$. Let us introduce new 
dimensionless variables:

$$
\begin{aligned}
s & =\left(2 R T_{0}\right)^{1 / 2} t / \ell, y=X_{1} /\left[t\left(2 R T_{0}\right)^{1 / 2}\right], \\
\eta_{i} & =\left[\xi_{i}-\left(X_{1} / t\right) \delta_{i 1}\right] /\left(2 R T_{0}\right)^{1 / 2},
\end{aligned}
$$

and rewrite Eqs. (1)-(3) in terms of the following variables:

$$
\begin{aligned}
G(s, y, \boldsymbol{\eta}) & =\rho_{0}^{-1}\left(2 R T_{0}\right)^{3 / 2} f\left(t, X_{1}, \boldsymbol{\xi}\right), \hat{\rho}(s, y)=\rho / \rho_{0}, \\
\hat{u}(s, y) & =u /\left(2 R T_{0}\right)^{1 / 2}, \hat{T}(s, y)=T / T_{0}, \hat{p}(s, y)=p / p_{0}, \\
\hat{p}_{\|}(s, y) & =p_{\|} / p_{0}, \hat{p}_{\perp}(s, y)=p_{\perp} / p_{0}, \hat{T}_{\|}(s, y)=T_{\|} / T_{0}, \\
\hat{T}_{\perp}(s, y) & =T_{\perp} / T_{0}, \hat{u}_{p}=u_{p} /\left(2 R T_{0}\right)^{1 / 2}, \\
\hat{a}_{0} & =a_{0} /\left(2 R T_{0}\right)^{1 / 2}=\sqrt{\gamma / 2}=\sqrt{5 / 6} .
\end{aligned}
$$

Here, $\ell=\left(2 R T_{0}\right)^{1 / 2} /\left(A_{\mathrm{c}} \rho_{0}\right)$ is the mean free path of a molecule at a resting equilibrium state with temperature $T_{0}$ and mass density $\rho_{0}, p_{0}=\rho_{0} R T_{0}$ is a reference pressure, and the independent variables have been changed from $\left(t, X_{1}, \xi\right)$ into $(s, y, \eta)$. The use of this set of variables is particularly advantageous for the analysis of the long time behavior, in which $s$ (or $t$ ) and thus $X_{1}$ become indefinitely large. After the transformation, we obtain for $G$ the following equation

$$
\begin{aligned}
& s \partial_{s} G+\eta_{1}\left(\partial_{y} G-\partial_{\eta_{1}} G\right) \\
& \quad=s \hat{\rho}(\mathcal{G}-G),\left(0<y<\hat{u}_{p}\right), \\
& \mathcal{G}=\frac{\hat{\rho}}{(\pi \hat{T})^{3 / 2}} \exp \left[-\frac{\left(\eta_{1}-v\right)^{2}+\eta_{2}^{2}+\eta_{3}^{2}}{\hat{T}}\right], \\
& \hat{\rho}=\int G d \eta, \quad \hat{\rho} v=\int \eta_{1} G d \eta, \\
& \hat{p}=\hat{\rho} \hat{T}=\frac{2}{3} \int\left[\left(\eta_{1}-v\right)^{2}+\eta_{2}^{2}+\eta_{3}^{2}\right] G d \eta,
\end{aligned}
$$

the boundary conditions

$$
\begin{aligned}
G & =\mathcal{G}_{A},\left(y=0, \eta_{1}>0\right), \\
G\left(\eta_{1}\right) & =G\left(-\eta_{1}\right),\left(y=\hat{u}_{p}, \eta_{1}<0\right),
\end{aligned}
$$

and the initial condition

$$
G=G_{0} \equiv \begin{cases}\mathcal{G}_{A}, & \left(\eta_{1}>0\right), \\ \mathcal{G}_{B}, & \left(\eta_{1}<0\right) .\end{cases}
$$

Here,

$$
\begin{aligned}
\mathcal{G}_{A} & =\pi^{-3 / 2} \exp \left(-\left[\left(\eta_{1}-v_{0}\right)^{2}+\eta_{2}^{2}+\eta_{3}^{2}\right]\right), \\
\mathcal{G}_{B} & =\mathcal{G}_{A}\left(\eta_{1}-2\left(\eta_{1}-v_{p}\right), \eta_{2}, \eta_{3}\right), \\
v_{p} & =\hat{u}_{p}-y, \quad v_{0}=\hat{a}_{0}-y .
\end{aligned}
$$

It should be noted that the dimensionless flow velocity $\hat{u}$ is recovered from $v$ by the relation $\hat{u}=v+y$. In what follows, we call $v$ the (dimensionless) pseudo flow velocity. Dimensionless parallel and perpendicular pressure and temperature are given by

$$
\begin{aligned}
& \hat{p}_{\|}=\hat{\rho} \hat{T}_{\|}=2 \int\left(\eta_{1}-v\right)^{2} G d \eta, \\
& \hat{p}_{\perp}=\hat{\rho} \hat{T}_{\perp}=\int\left(\eta_{2}^{2}+\eta_{3}^{2}\right) G d \eta .
\end{aligned}
$$

\section{PRELIMINARY}

We summarize main results of the isentropic flow theory in the conventional gas dynamics and those of the kinetic theory in the free molecular limit. In both cases, the macroscopic quantities are self-similar; namely, they depend on $\left(t, X_{1}\right)$ [or $(s, y)]$ as a function of a single variable $X_{1} / t$ (or $y$ ). However, apart from this, the gas behavior is remarkably different, as we show below.

\section{A. Isentropic flow theory of conventional gas dynamics}

Consider a propagation of an expansion wave toward a uniform upstream with pressure $p_{U}$, temperature $T_{U}$, and flow velocity $\left(u_{U}, 0,0\right)$. According to the isentropic flow theory of the conventional gas dynamics [1], the pressure $p$ and temperature $T$ at the position with flow velocity $(u, 0,0)$ are given by

$$
\begin{aligned}
\frac{p}{p_{U}} & =\left(1-\frac{\gamma-1}{2} \frac{u-u_{U}}{\sqrt{\gamma R T_{U}}}\right)^{\frac{2 \gamma}{\gamma-1}}, \\
\frac{T}{T_{U}} & =\left(1-\frac{\gamma-1}{2} \frac{u-u_{U}}{\sqrt{\gamma R T_{U}}}\right)^{2} .
\end{aligned}
$$

We apply this formula to the present situation where the upstream condition is given by $p_{U}=p_{0}, T_{U}=T_{0}$, and $u_{U}=$ $a_{0}$, bearing in mind that the expansion wave is generated when $u_{p}>a_{0}$ and that the expansion front does not move. Inside the expansion wave, the pressure and temperature vary (actually decrease) in $X_{1} / t$ (or $y$ ) in accordance with Eq. (11) from their upstream values to the values at the tail:

$$
\begin{aligned}
& \frac{p_{p}}{p_{0}}=\left(\frac{\gamma+1}{2}-\frac{\gamma-1}{2} \frac{u_{p}}{a_{0}}\right)^{\frac{2 \gamma}{\gamma-1}}, \\
& \frac{T_{p}}{T_{0}}=\left(\frac{\gamma+1}{2}-\frac{\gamma-1}{2} \frac{u_{p}}{a_{0}}\right)^{2} .
\end{aligned}
$$

Therefore, when $\left(a_{0}<\right) u_{p}<u_{c} \equiv \frac{\gamma+1}{\gamma-1} a_{0}$, the downstream of the expansion wave does not reach the vacuum state but is connected to a uniform state with pressure $p_{p}$, temperature $T_{p}$, and flow velocity $\left(u_{p}, 0,0\right)$. When $u_{p}>u_{c}\left(=\frac{\gamma+1}{\gamma-1} a_{0}=4 a_{0}\right)$, on the other hand, the gas pressure reaches zero at the tail, forming a vacuum region behind it. The location of the tail in the case of $u_{p} \leqslant u_{c}$ is determined as $y_{\text {tail }}=\frac{\gamma+1}{2}\left(\hat{u}_{p}-\hat{a}_{0}\right)$ from Eq. (13) that appears later by setting $\hat{u}=\hat{u}_{p}$.

The profile of the expansion wave in $y$ can be determined in the following way. Since the propagation of a wave front with flow velocity $u$ is determined [1] by $\frac{d X_{1}}{d t}=\frac{\gamma+1}{2}\left(u-a_{0}\right)$, we obtain

$$
\hat{u}=\frac{2}{\gamma+1} y+\hat{a}_{0}
$$

which yields by substitution into Eq. (11)

$$
\hat{p}(=\hat{\rho} \hat{T})=\left(1-\frac{y}{\hat{u}_{c}}\right)^{\frac{2 \gamma}{\gamma-1}}, \quad \hat{T}=\left(1-\frac{y}{\hat{u}_{c}}\right)^{2} .
$$

Here, $\hat{u}_{c}=\frac{\gamma+1}{\gamma-1} \hat{a}_{0}=4 \hat{a}_{0}$. It should be noted that, since $y_{\text {tail }}\left[=\frac{\gamma+1}{2}\left(\hat{u}_{p}-\hat{a}_{0}\right)\right]=\hat{u}_{p}+\frac{\gamma-1}{2}\left(\hat{u}_{p}-\hat{u}_{c}\right)$, the location of the expansion tail is upstream of the piston when the piston velocity $u_{p}$ is less than the critical velocity $u_{c}$. When $u_{p}=u_{c}$, 


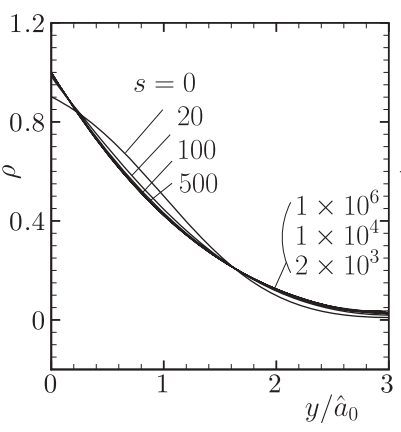

(a)

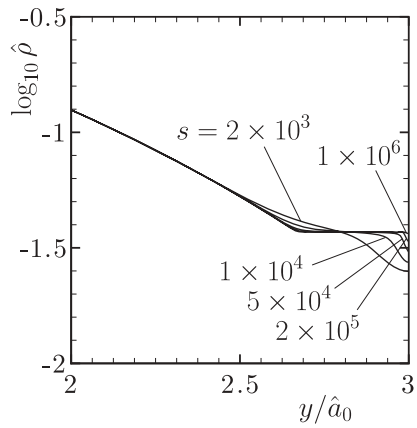

(e)

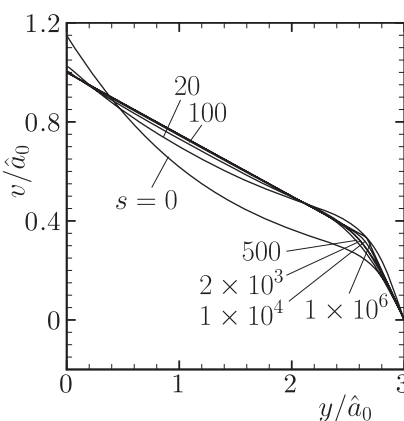

(b)

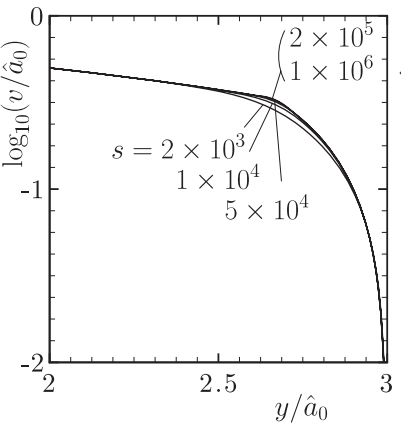

(f)

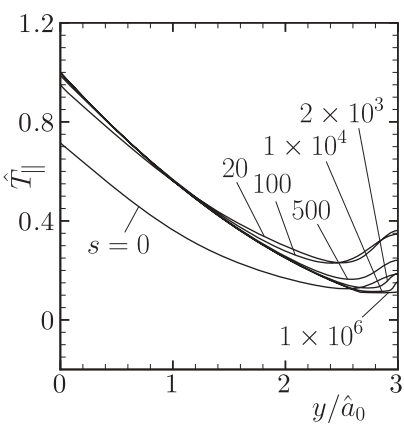

(c)

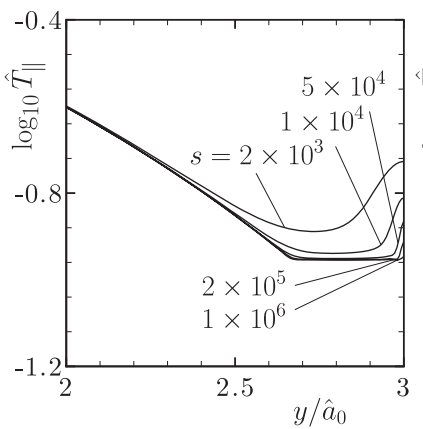

(g)

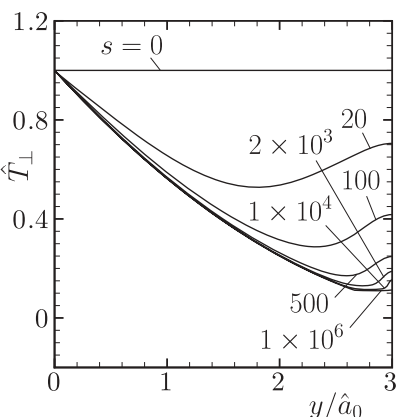

(d)

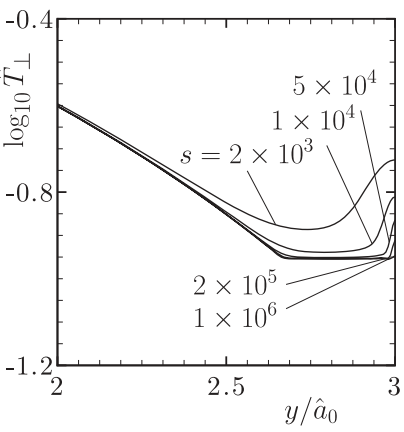

(h)

FIG. 3. Profiles of the macroscopic quantities for various values of $s$ : I $\left(u_{p} / a_{0}=3\right.$ ). (a) $\rho / \rho_{0}$ vs $y$, (b) $v / \hat{a}_{0}$ vs $y$, (c) $T_{\|} / T_{0}$ vs $y$, and (d) $T_{\perp} / T_{0}$ vs $y$. Panels (e)-(h) are the semilog versions of panels (a)-(d) in the interval $2 \leqslant y / \hat{a}_{0} \leqslant 3$.

the tail position coincides with the piston position, namely, $y_{\text {tail }}=\hat{u}_{p}\left(=\hat{u}_{c}\right)$. When $u_{p}$ exceeds the critical velocity $u_{c}$,

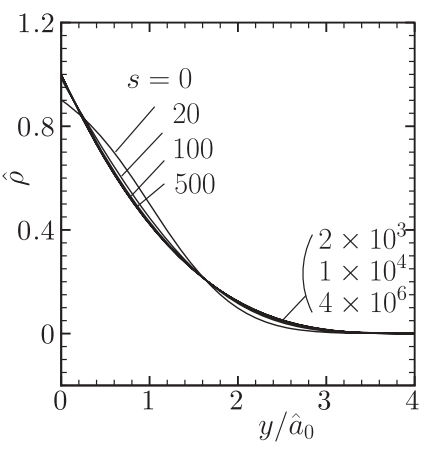

(a)

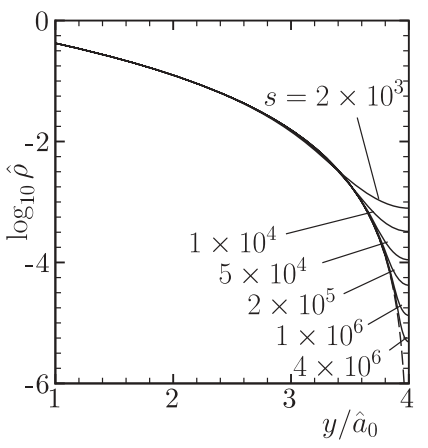

(e)

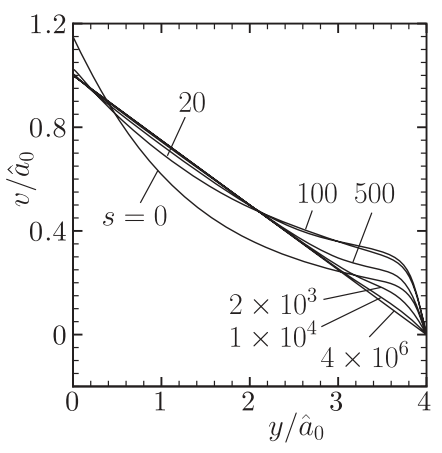

(b)

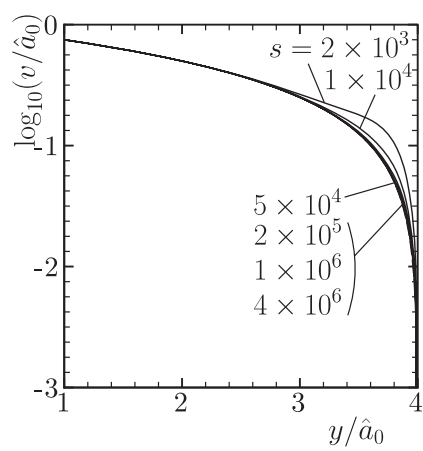

(f)

Eq. (14) is valid only for $y<\hat{u}_{c}$, and the vacuum region appears for $y>\hat{u}_{c}$; thus, the tail location is again $y_{\text {tail }}=\hat{u}_{c}$.

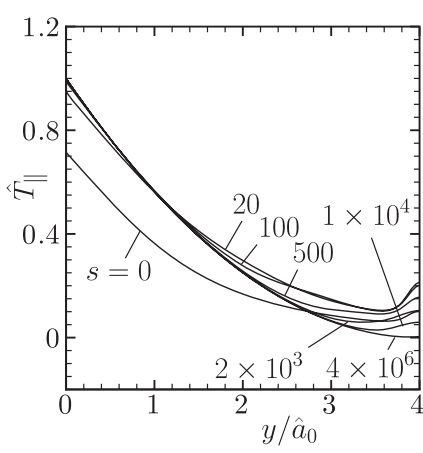

(c)

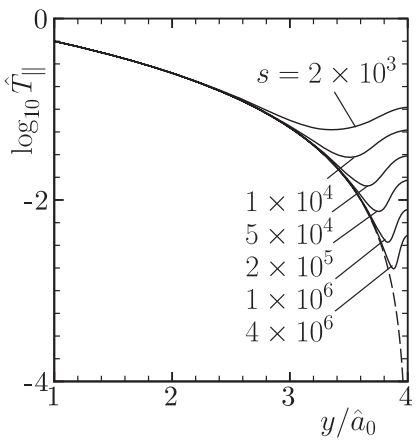

(g)

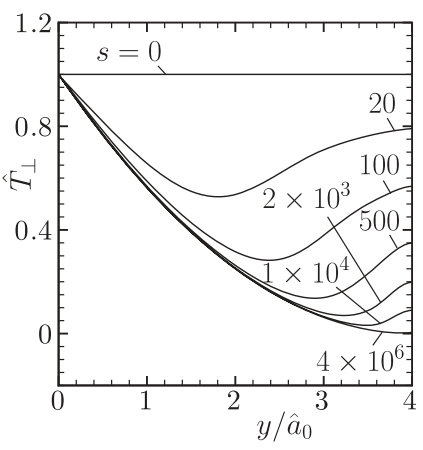

(d)

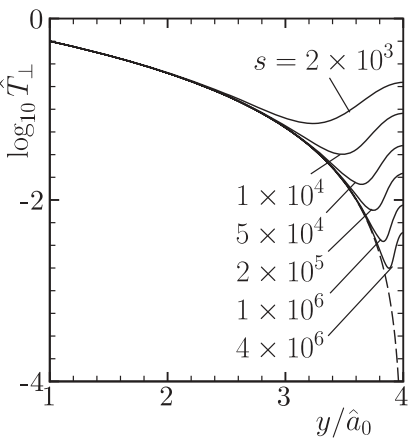

(h)

FIG. 4. Profiles of the macroscopic quantities for various values of $s$ : II $\left(u_{p} / a_{0}=4\right)$. (a) $\rho / \rho_{0}$ vs $y$, (b) $v / \hat{a}_{0}$ vs $y$, (c) $T_{\|} / T_{0}$ vs $y$, and (d) $T_{\perp} / T_{0}$ vs $y$. Panels (e)-(h) are the semilog versions of panels (a)-(d) in the interval $1 \leqslant y / \hat{a}_{0} \leqslant 4$. Dashed lines indicate the solution of the expansion wave (14) in the conventional gas dynamics. 


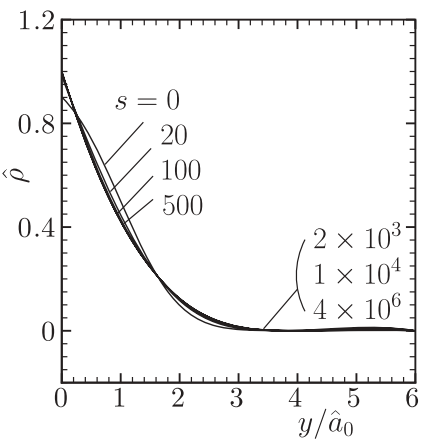

(a)

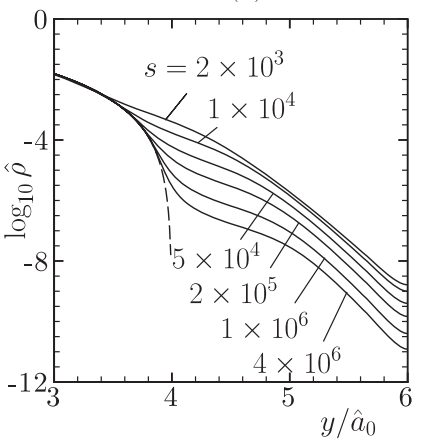

(e)

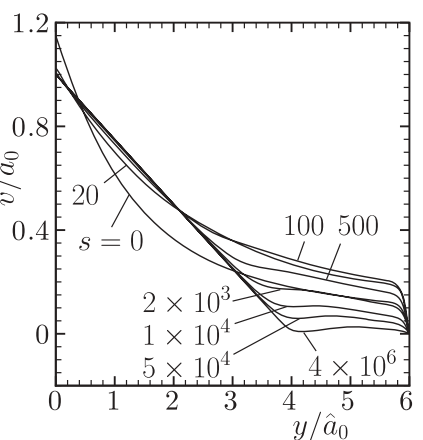

(b)

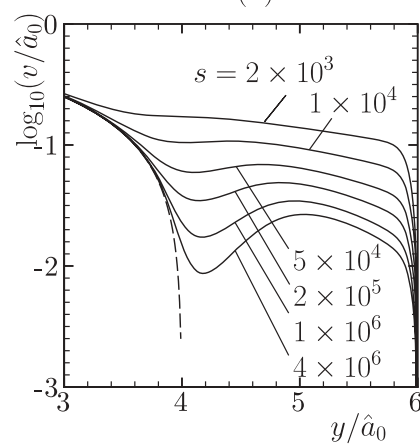

(f)

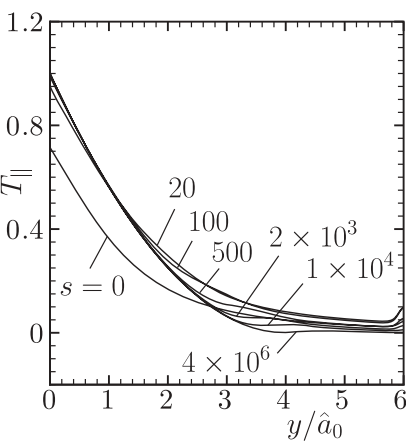

(c)

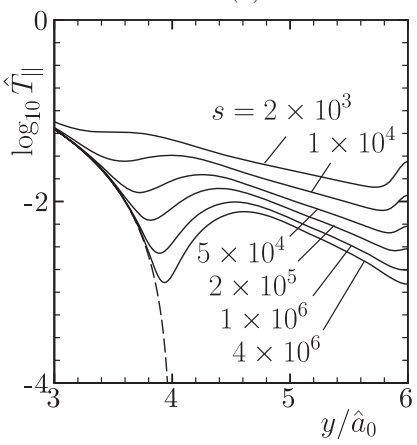

(g)

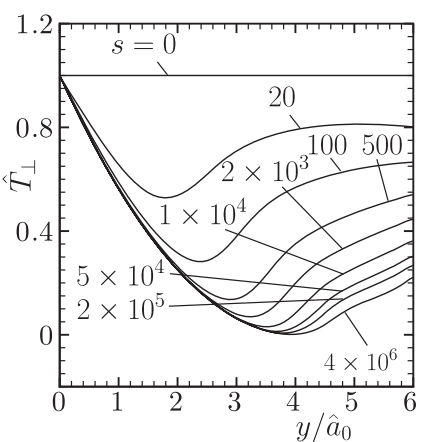

(d)

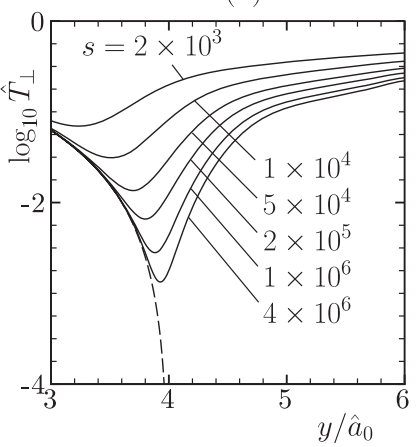

(h)

FIG. 5. Profiles of the macroscopic quantities for various values of $s$ : III $\left(u_{p} / a_{0}=6\right.$ ). (a) $\rho / \rho_{0}$ vs $y$, (b) $v / \hat{a}_{0}$ vs $y$, (c) $T_{\|} / T_{0}$ vs $y$, and (d) $T_{\perp} / T_{0}$ vs $y$. Panels (e)-(h) are the semilog versions of panels (a)-(d) in the interval $3 \leqslant y / \hat{a}_{0} \leqslant 6$. Dashed lines indicate the solution of the expansion wave (14) in the conventional gas dynamics.

It should be mentioned that the local Maxwellian corresponding to the expansion wave is not a solution of the Boltzmann (or its model) equation [10] but rather its asymptotic limit for $0<y<y_{\text {tail }}$ when $s \rightarrow \infty$. This will be demonstrated numerically in Sec. VI. Moreover, in Appendix A, by assuming a nonvanishing mass density, we analytically show that a slowly varying solution for large $s$ such that $s \partial_{s} G=O(G)$ when $s \gg 1$ asymptotically approaches that Maxwellian. A corresponding mathematical argument can be found in Ref. [17] in the case of a more simplified kinetic equation.

\section{B. Free molecular limit}

Because $f_{0}$ satisfies boundary conditions (2a) and (2b), it (or $G_{0}$ ) is a solution of the problem, Eqs. (1)-(3) [or Eqs. (6)-(8)], in the free molecular limit; thus the macroscopic quantities in this limit are immediately obtained as

$$
\begin{aligned}
\hat{\rho}= & 1-\frac{1}{2}\left[\operatorname{erf}\left(y_{*}\right)+\operatorname{erf}\left(y_{\dagger}\right)\right], \\
\hat{\rho} v= & \frac{1}{2 \sqrt{\pi}}\left[e^{-y_{*}^{2}}-e^{-y_{\dagger}^{2}}\right]+\left(\hat{u}_{p}-y\right)+\frac{1}{2}\left[y_{*} \operatorname{erf}\left(y_{*}\right)\right. \\
& \left.-y_{\dagger} \operatorname{erf}\left(y_{\dagger}\right)\right], \\
\hat{p}_{\|}\left(=\hat{\rho} \hat{T}_{\|}\right)= & \hat{\rho}\left[\left(1-2 v^{2}\right)+2 y_{*}^{2}\right]+4\left(\hat{u}_{p}-y\right)\left(\hat{u}_{p}-\hat{a}_{0}\right) \\
& \times\left[1-\operatorname{erf}\left(y_{\dagger}\right)\right]-\frac{1}{\sqrt{\pi}}\left(y_{*} e^{-y_{*}^{2}}+y_{\dagger} e^{-y_{\dagger}^{2}}\right),
\end{aligned}
$$

$\hat{p}_{\perp}\left(=\hat{\rho} \hat{T}_{\perp}\right)=1-\frac{1}{2}\left[\operatorname{erf}\left(y_{*}\right)+\operatorname{erf}\left(y_{\dagger}\right)\right]=\hat{\rho}$.
Here $\quad y_{*}=y-\hat{a}_{0}, \quad y_{\dagger}=2 \hat{u}_{p}-\hat{a}_{0}-y, \quad$ and $\quad \operatorname{erf}(x)=$ $(2 / \sqrt{\pi}) \int_{0}^{x} \exp \left(-t^{2}\right) d t$.

Note that the quantities are independent of $\hat{u}_{c}$ and that $\hat{T}_{\perp}$ is identically unity but $\hat{T}_{\|}$varies in $y$; in other words, the temperature is anisotropic (i.e., $\hat{T}_{\perp} \neq \hat{T}_{\|}$). Since $y<\hat{u}_{p}$, the mass density does not vanish as far as the piston speed is finite. Hence, no vacuum region appears for finite $\hat{u}_{p}$. In the limit $\hat{u}_{p} \rightarrow \infty$, we obtain

$$
\begin{aligned}
\hat{\rho} & \rightarrow \frac{1}{2}\left[1-\operatorname{erf}\left(y_{*}\right)\right], v \rightarrow \frac{1}{2 \sqrt{\pi} \hat{\rho}} e^{-y_{*}^{2}}-y_{*}, \\
\hat{T}_{\|} & \rightarrow\left(1-2 v^{2}\right)+2 y_{*}^{2}-\frac{y_{*} e^{-y_{*}^{2}}}{\sqrt{\pi} \hat{\rho}},
\end{aligned}
$$

all of which vanish as $y \rightarrow \infty$. Therefore, the vacuum region may appear only in the far field of $y$ when the piston speed is infinite. Since $\hat{T}_{\perp}$ is identically unity, the temperature becomes highly anisotropic and $\hat{T} \rightarrow 2 / 3$ as $\left(\hat{u}_{p}, y\right) \rightarrow \infty$. In this way, the free molecular gas behaves quite differently from the continuum gas described by the conventional gas dynamics.

\section{NUMERICAL RESULTS}

The initial- and boundary-value problem, Eqs. (6)-(8), in Sec. III can be reduced to that for marginal distribution 


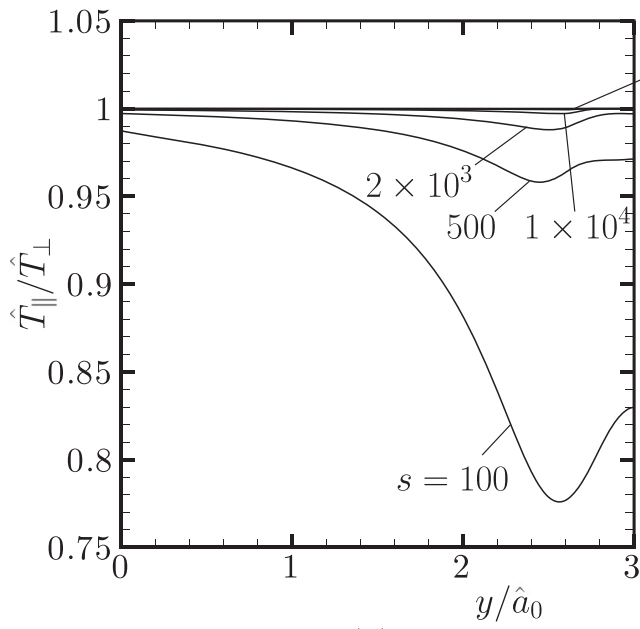

(a)

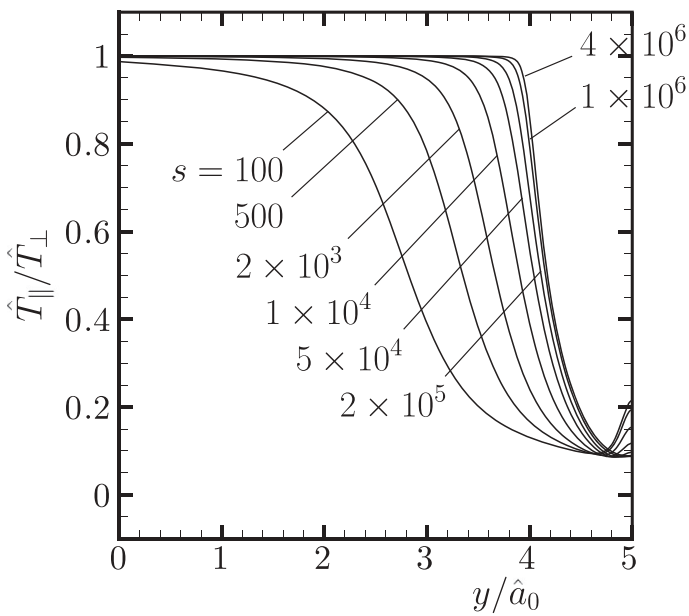

(c)

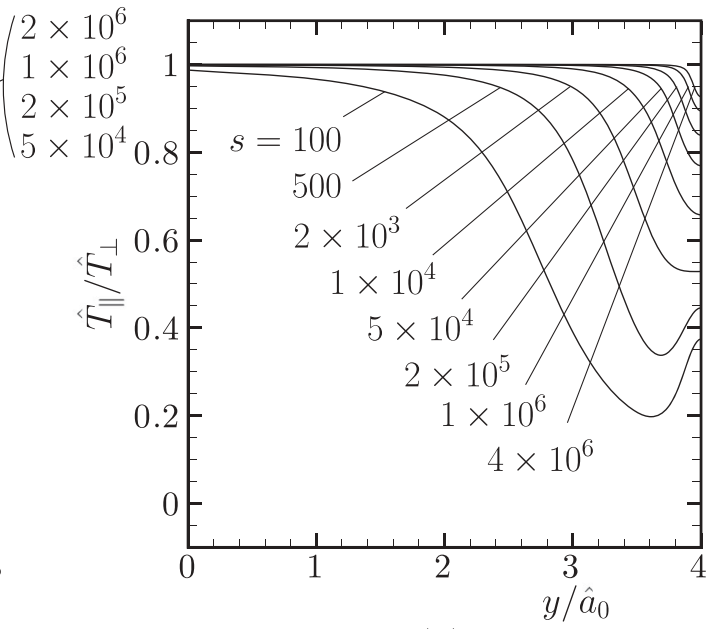

(b)

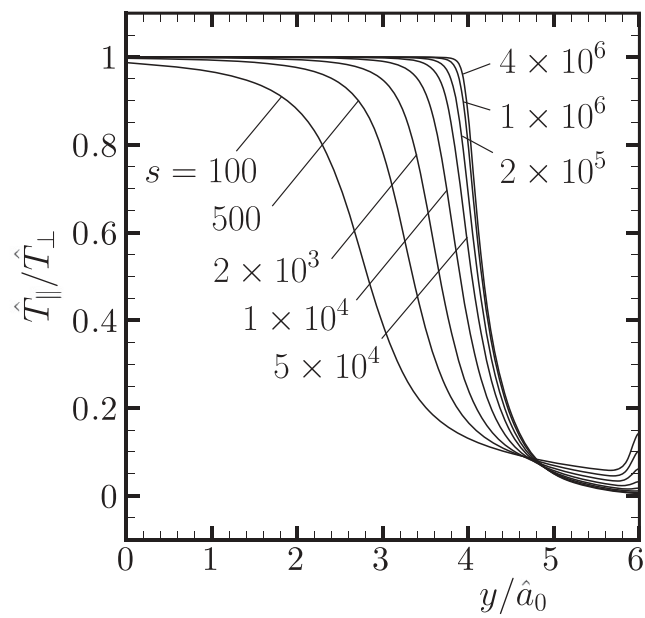

(d)

FIG. 6. Profile of $T_{\|} / T_{\perp}$ for various values of $s$. (a) $u_{p} / a_{0}=3$, (b) $u_{p} / a_{0}=4$, (c) $u_{p} / a_{0}=5$, and (d) $u_{p} / a_{0}=6$.

functions (Chu's method):

$$
\begin{aligned}
& g\left(s, y, \eta_{1}\right)=\int G(s, y, \eta) d \eta_{2} d \eta_{3}, \\
& h\left(s, y, \eta_{1}\right)=\int\left(\eta_{2}^{2}+\eta_{3}^{2}\right) G(s, y, \eta) d \eta_{2} d \eta_{3},
\end{aligned}
$$

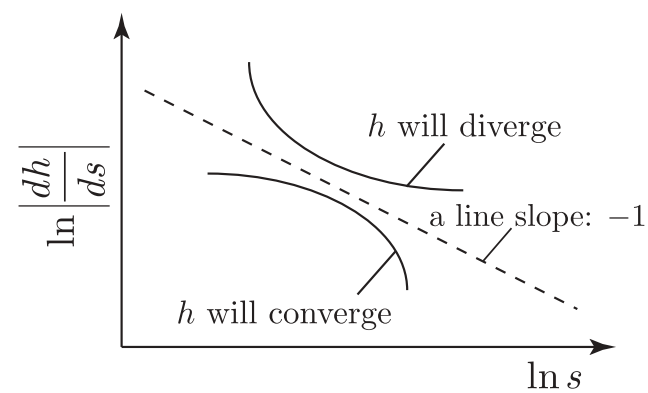

FIG. 7. Sketch of the prediction method for the large time behavior. If the double logarithmic plot of $d h / d s$ vs $s$ is bounded by a line with slope -1 from above (below), the quantity $h$ will converge (diverge) as $s \rightarrow \infty$. which we solve numerically by a splitting method. The details of the method are summarized in Appendix B, together with the data of computations.

Numerical computations are done for $u_{p} / a_{0}=3,4,5$, and 6. According to the conventional gas dynamics (CGD), the gas density remains finite for $u_{p} / a_{0}=3$, it decreases in $y$ and vanishes at the piston for $u_{p} / a_{0}=4(=$ $\left.u_{c} / a_{0}\right)$, and the vacuum region appears between the expansion tail and the piston for $u_{p} / a_{0}=5$ and 6 (see Fig. 2).

Figure 3 shows the profiles of $\rho / \rho_{0}, v / \hat{a}_{0}, T_{\|} / T_{0}$, and $T_{\perp} / T_{0}$ for various values of $s$ in the case of $u_{p} / a_{0}=3$. At $s=$ $1 \times 10^{6}$, the profiles have almost converged to those predicted by the conventional gas dynamics (CGD profiles, for short). The parallel and perpendicular temperatures stay different from the CGD profiles for a long time duration, compared with the other quantities. For $s \gtrsim 2000$, the profiles of parallel and perpendicular temperatures are almost identical (isotropic temperature). Figure 4 shows the profiles of $\rho / \rho_{0}, v / \hat{a}_{0}, T_{\|} / T_{0}$, and $T_{\perp} / T_{0}$ in the critical case $u_{p} / a_{0}=4$. Again, the profiles approach the CGD profiles. However, the temperature remains 


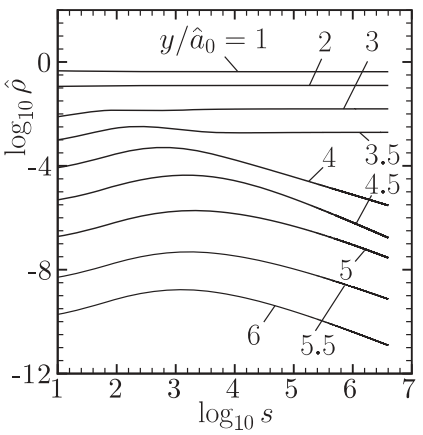

(a)

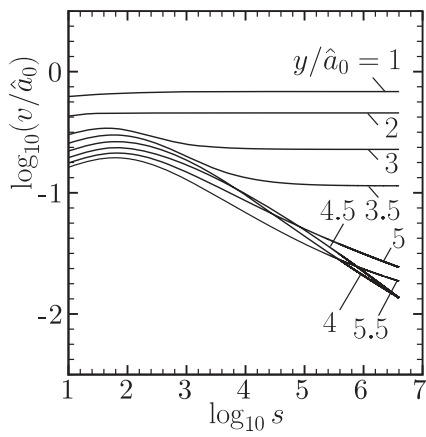

(b)

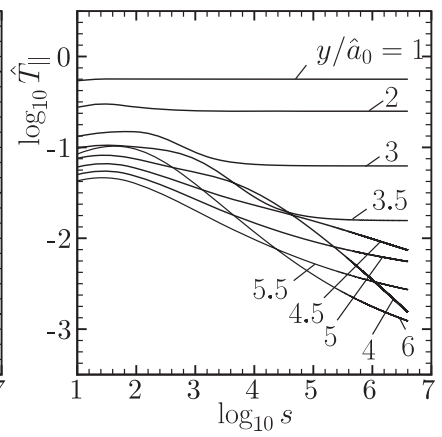

(c)

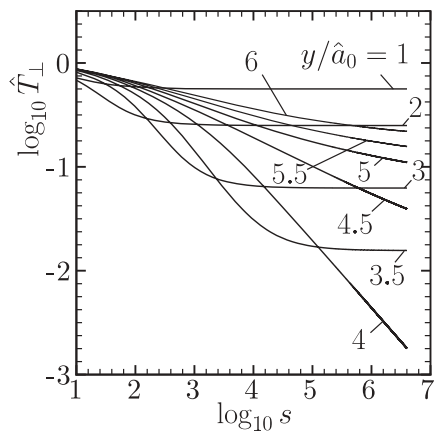

(d)

FIG. 8. Log-log plots of the time evolution of the macroscopic variables for various values of $y\left(u_{p} / a_{0}=6\right)$. (a) $\rho / \rho_{0}$ vs $s$, (b) $v / \hat{a}_{0}$ vs $s$, (c) $T_{\|} / T_{0}$ vs $s$, and (d) $T_{\perp} / T_{0}$ vs $s$.

anisotropic for a long time, especially near the expansion tail, compared with the previous case.

Figure 5 shows the profiles of $\rho / \rho_{0}, v / \hat{a}_{0}, T_{\|} / T_{0}$, and $T_{\perp} / T_{0}$ for various values of $s$ in the case of $u_{p} / a_{0}=6$. As time goes on, the profiles approach those of the expansion wave for $y / \hat{a}_{0}<4\left(=\hat{u}_{c}\right)$. For $y / \hat{a}_{0}>4$, which corresponds to the vacuum region in CGD, each quantity decreases in time but its profile is not monotonically decreasing in $y$ except for $\rho / \rho_{0}$; in particular, $T_{\perp} / T_{0}$ increases as $y$ increases and the temperature is anisotropic. Therefore, as time goes on, the gas approaches the local equilibrium for $y / \hat{a}_{0}<4$ but remains out of equilibrium for $y / \hat{a}_{0}>4$. The qualitative features described here also apply to the case of $u_{p} / a_{0}=5$.

In this way, the behavior of the gas undergoes a dramatic change in quality at around $y / \hat{a}_{0}=4$. To demonstrate this more clearly, we show in Fig. 6 the profile of the temperature ratio $T_{\|} / T_{\perp}$ for various values of $s$ in the cases of $u_{p} / a_{0}=$ $3,4,5$, and 6 . Since the ratio is unity for the (local) Maxwellian, its deviation from unity is a measure of nonequilibrium. It is seen that, as time goes on, $T_{\|} / T_{\perp}$ approaches unity for $y / \hat{a}_{0}<4$, while it approaches a smaller positive value at each point of $y$ for $y / \hat{a}_{0}>4$. Moreover, this value decreases as $y$ increases. This means that in the region $y / \hat{a}_{0}>4$, the intermolecular collisions do not occur sufficiently, so that the gas remains out of equilibrium even after an infinitely long time has passed. The degree of nonequilibrium becomes higher for larger $y$. It is also seen from a comparison between
Figs. 6(c) and 6(d) that a higher nonequilibrium region appears for larger $u_{p} / a_{0}$.

\section{NUMERICAL PREDICTION ABOUT THE LONG TIME LIMIT}

The main features of the flows in the case of $u_{p} / a_{0}>4$ described in Sec. V (see Figs. 5 and 6) are as follows.

(1) For $0<y / \hat{a}_{0}<4$, the gas state approaches the local Maxwellian corresponding to the expansion wave in the conventional gas dynamics.

(2) For $y / \hat{a}_{0}>4$, all of $\rho / \rho_{0}, v, T_{\|} / T_{0}$, and $T_{\perp} / T_{0}$ decrease in time; $\rho / \rho_{0}$ decreases faster than the others, while $T_{\perp} / T_{0}$ decreases much slower than the others and remains appreciable even at $s=4 \times 10^{6}$. The ratio $T_{\|} / T_{\perp}$ largely deviates from unity, meaning strong nonequilibrium.

We examine the data more closely and discuss the behavior of the gas as $s \rightarrow \infty$ for $u_{p} / a_{0}>4$.

\section{A. Method of numerical prediction}

To analyze the tendency of time evolution of a quantity $h(s, y)$, we sample the sequence $h_{n}$ from the numerical data for a fixed $y$ from a certain instance $s_{0}$ with a uniform interval $\sigma$, namely, $h_{n}=h\left(s_{n}, \cdot\right)\left(s_{n}=s_{0}+n \sigma ; n=0, \ldots, N\right)$, and make the double logarithmic plots as depicted in Fig. 7. Here,

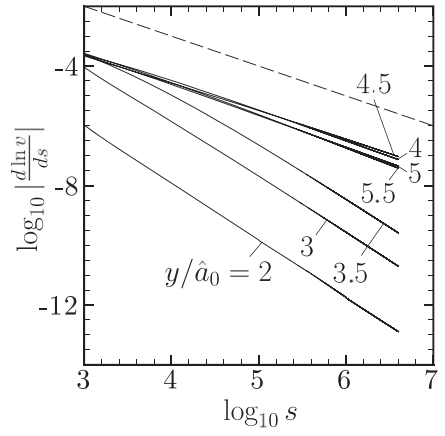

(a)

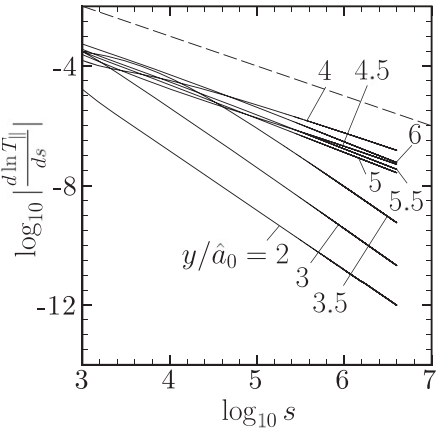

(b)

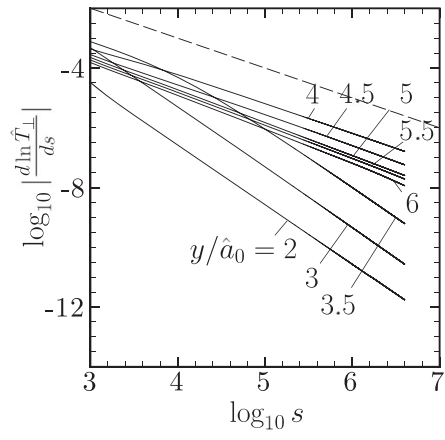

(c)

FIG. 9. Log-log plots of the change rate in time of the macroscopic quantities for various values of $y\left(u_{p} / a_{0}=6\right)$. (a) $d \ln v / d s$ vs $s$, (b) $d \ln \left(T_{\|} / T_{0}\right) / d s$ vs $s$, and (c) $d \ln \left(T_{\perp} / T_{0}\right) / d s$ vs $s$. Dashed lines indicate a line with slope -1 . 
TABLE I. $F[\hat{\rho}], F[v], F\left[\hat{T}_{\|}\right]$, and $F\left[\hat{T}_{\perp}\right]$ for several values of $s\left(u_{p} / a_{0}=6\right)$.

\begin{tabular}{|c|c|c|c|c|c|c|c|c|c|c|c|}
\hline \multirow[b]{2}{*}{$s \backslash$} & \multirow[b]{2}{*}{$y / \hat{a}_{0}$} & \multicolumn{5}{|c|}{$-F[\hat{\rho}]$} & \multicolumn{5}{|c|}{$-F[v]$} \\
\hline & & 4.0 & 4.5 & 5.0 & 5.5 & 6.0 & 4.0 & 4.5 & 5.0 & 5.5 & 6.0 \\
\hline $2 \times 10^{5}$ & & 0.996 & 0.935 & 0.879 & 0.907 & 0.925 & 1.030 & 0.953 & 1.062 & 1.115 & - \\
\hline $5 \times 10^{5}$ & & 0.998 & 0.960 & 0.912 & 0.933 & 0.948 & 1.041 & 0.965 & 1.060 & 1.126 & - \\
\hline $1 \times 10^{6}$ & & 0.998 & 0.972 & 0.929 & 0.947 & 0.961 & 1.045 & 0.970 & 1.057 & 1.130 & - \\
\hline $2 \times 10^{6}$ & & 0.996 & 0.981 & 0.944 & 0.959 & 0.973 & 1.044 & 0.973 & 1.053 & 1.127 & - \\
\hline \multirow[t]{2}{*}{$4 \times 10^{6}$} & & 0.994 & 0.986 & 0.956 & 0.969 & 0.986 & 1.038 & 0.970 & 1.050 & 1.117 & - \\
\hline & & \multicolumn{5}{|c|}{$-F\left[\hat{T}_{\|}\right]$} & \multicolumn{5}{|c|}{$-F\left[\hat{T}_{\perp}\right]$} \\
\hline$s \backslash$ & $y / \hat{a}_{0}$ & 4.0 & 4.5 & 5.0 & 5.5 & 6.0 & 4.0 & 4.5 & 5.0 & 5.5 & 6.0 \\
\hline $2 \times 10^{5}$ & & 0.925 & 0.942 & 1.142 & 1.139 & 1.150 & 0.993 & 1.033 & 1.111 & 1.115 & 1.145 \\
\hline $5 \times 10^{5}$ & & 0.947 & 0.968 & 1.136 & 1.141 & 1.163 & 0.996 & 1.050 & 1.103 & 1.112 & 1.141 \\
\hline $1 \times 10^{6}$ & & 0.961 & 0.987 & 1.130 & 1.139 & 1.166 & 1.001 & 1.060 & 1.100 & 1.117 & 1.159 \\
\hline $2 \times 10^{6}$ & & 0.975 & 1.006 & 1.129 & 1.136 & 1.158 & 1.006 & 1.069 & 1.110 & 1.144 & 1.254 \\
\hline $4 \times 10^{6}$ & & 0.986 & 1.020 & 1.140 & 1.142 & 1.135 & 1.010 & 1.070 & 1.115 & 1.163 & 1.281 \\
\hline
\end{tabular}

$d h / d s$ is evaluated by a simple finite difference:

$$
\left.\frac{d h}{d s}\right|_{s=s_{i}} \sim \frac{h_{i}-h_{i-1}}{\sigma}, \quad i=1, \ldots, N
$$

If the curve connecting the sequence points is bounded from above by a line with slope less than $-1, h$ should converge as $s \rightarrow \infty$. If the curve is bounded from below by a line with slope -1 or larger, $h$ diverges.

We apply this method to $h=\hat{\rho}, v, \hat{T}_{\|}, \hat{T}_{\perp}$, and $T_{\|} / T_{\perp}$ and estimate the asymptotic limit for $s \rightarrow \infty$ by extrapolating the curve using its tangential line at $s=s_{*}$ in the double logarithmic plots. If the curve is nearly linear, the estimated value is little influenced by the choice of $s_{*}$ and is close to the limiting value as $s \rightarrow \infty$. If the curve is upwards convex, using larger $s_{*}$ yields a better estimate. We also apply the same method to the sequence $\left\{y_{n}\right\}$ generated by the relation $c=T_{\|} / T_{\perp}\left(s_{n}, y_{n}\right)$ for a certain constant $c$ to estimate $y$ such that $c=\lim _{s \rightarrow \infty} T_{\|} / T_{\perp}(s, y)$.

Furthermore, in order to judge whether $\hat{\rho}, v, \hat{T}_{\|}$, and $\hat{T}_{\perp}$ for a fixed $y$ remain finite or vanish as $s \rightarrow \infty$, the above method is applied to $h=\ln \hat{\rho}, \ln v, \ln \hat{T}_{\|}$, and $\ln \hat{T}_{\perp}$. The slopes of the curves are then given by $F[\hat{\rho}], F[v], F\left[\hat{T}_{\|}\right]$, and $F\left[\hat{T}_{\perp}\right]$, where $F[\phi]=d(\ln |d \ln \phi / d s|) / d \ln s$, and $\phi=\hat{\rho}, v, \hat{T}_{\|}, \hat{T}_{\perp}$ is judged to vanish as $s \rightarrow \infty$ when $F[\phi] \geqslant-1$.

\section{B. Asymptotic behavior of the numerical solution}

Figure 8 shows a time evolution of $\hat{\rho}\left(=\rho / \rho_{0}\right), v, \hat{T}_{\|}(=$ $\left.T_{\|} / T_{0}\right)$, and $\hat{T}_{\perp}\left(=T_{\perp} / T_{0}\right)$ for various values of $y$ in the case of $u_{p} / a_{0}=6$. The qualitative change of gas behavior at $y / \hat{a}_{0}=4$, already pointed out in Sec. $\mathrm{V}$, is clearly observed in the figure: the logarithm of each quantity approaches a certain constant for $y / \hat{a}_{0}<4$, while it decreases for $y / \hat{a}_{0} \geqslant 4$. Among the shown quantities, $\hat{\rho}$ exhibits a clear tendency to vanish as $s \rightarrow \infty$ for each value of $y / \hat{a}_{0} \geqslant 4$. The others also tend to vanish at $y / \hat{a}_{0}=4$. Their decaying rate becomes, however, somewhat smaller for $y / \hat{a}_{0} \gtrsim 4.5$ as time proceeds, and accordingly it is not clear whether they tend to vanish or not as $s \rightarrow \infty$. To see it closely, we show the double logarithmic plots explained in the last paragraph of Sec. VIA in Fig. 9, together with the numerical values of the slopes $F[\hat{\rho}], F[v]$, $F\left[\hat{T}_{\|}\right]$, and $F\left[\hat{T}_{\perp}\right]$ at $s=2 \times 10^{5}, 5 \times 10^{5}, 1 \times 10^{6}, 2 \times 10^{6}$, and $4 \times 10^{6}$ in Table I. The line with slope -1 is also shown in the figure. The data of $F[\hat{\rho}]$ in the table support the above judgment that $\hat{\rho}$ tends to vanish. On the other hand, the data of $F\left[\hat{T}_{\|}\right]$and $F\left[\hat{T}_{\perp}\right]$ show that $T_{\|} / T_{0}$ and $T_{\perp} / T_{0}$ do not vanish

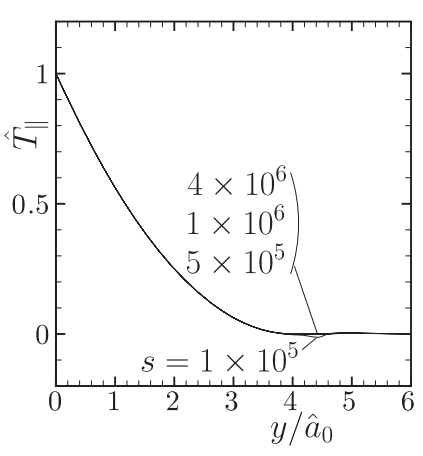

(a)

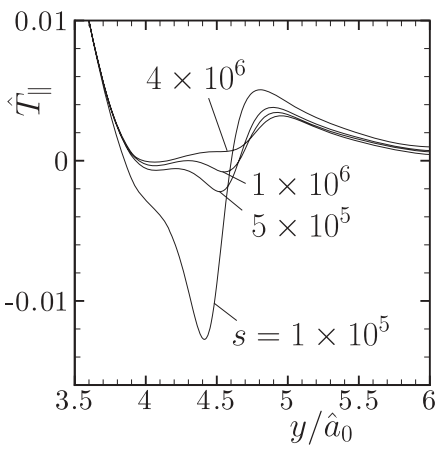

(b)

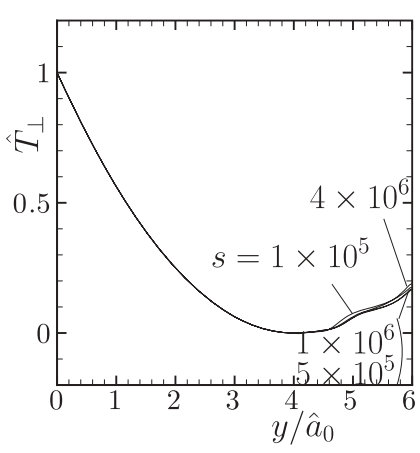

(c)

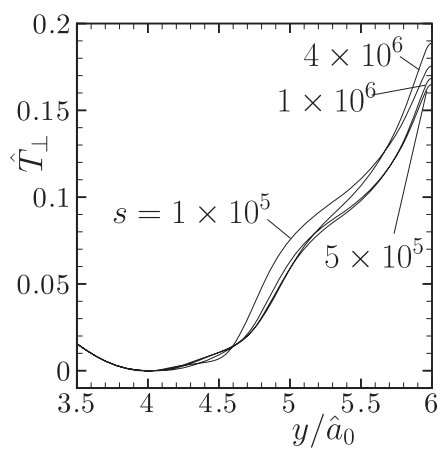

(d)

FIG. 10. Profiles of $T_{\|}$and $T_{\perp}$ in the limit $s \rightarrow \infty$ predicted at $s=1 \times 10^{5}, 5 \times 10^{5}, 1 \times 10^{6}$, and $4 \times 10^{6}\left(u_{p} / a_{0}=6\right.$ ). (a) $T_{\|} / T_{0}$ vs $y$, (b) the part $y / \hat{a}_{0}>3.5$ of (a) enlarged, (c) $T_{\perp} / T_{0}$ vs $y$, and (d) the part $y / \hat{a}_{0}>3.5$ of (c) enlarged. 


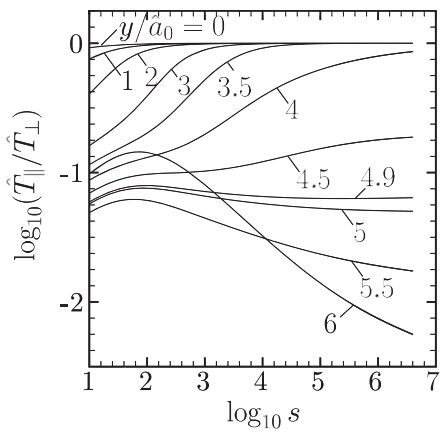

(a)

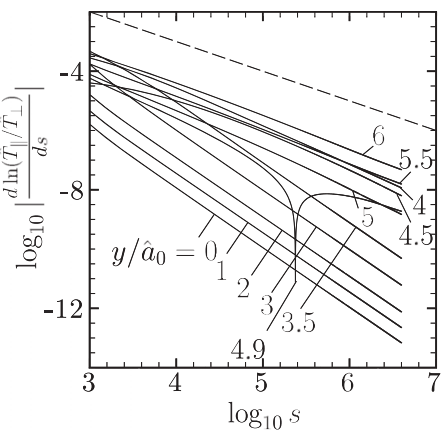

(b)

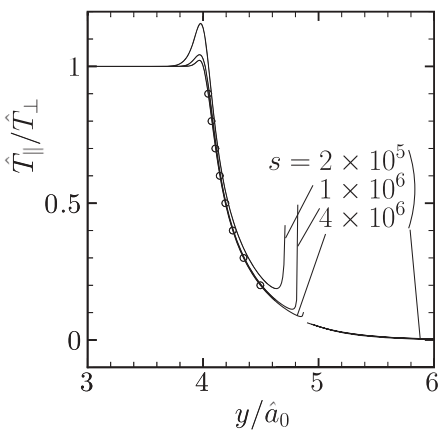

(c)

FIG. 11. Time evolution of $T_{\|} / T_{\perp}$ and its change rate for various values of $y$, and the profile of $T_{\|} / T_{\perp}$ in the limit $s \rightarrow \infty$ predicted at different values of $s\left(u_{p} / a_{0}=6\right.$ ). (a) $T_{\|} / T_{\perp}$ vs $s$, (b) $d \ln \left(T_{\|} / T_{\perp}\right) / d s$ vs $s$, and (c) $T_{\|} / T_{\perp}$ in the limit $s \rightarrow \infty$ predicted at $s=2 \times 10^{5}$, $1 \times 10^{6}$, and $4 \times 10^{6}$. The dashed line in (b) is a line with slope -1 . Solid lines in (c) indicate the predicted profile, except for the region where the prediction diverges. Open circles indicate the points $\left(y_{n}, 0.1 n\right)$ predicted at $s=4 \times 10^{6}$ such that $T_{\|} / T_{\perp}\left(s \rightarrow \infty, y_{n}\right)=0.1 n$, where $n=2,3, \ldots, 9$.

but remain finite (the frozen temperature) in the limit $s \rightarrow \infty$ for $y / \hat{a}_{0}>4$.

Figure 10 shows the profiles of $\hat{T}_{\|}$and $\hat{T}_{\perp}$ in the limit $s \rightarrow$ $\infty$ predicted at $s=1 \times 10^{5}, 5 \times 10^{5}, 1 \times 10^{6}$, and $4 \times 10^{6}$ by the method explained in Sec. VIA. The predicted profile $T_{\perp} / T_{0}$ for $y / \hat{a}_{0}>4$ appreciably deviates from zero, meaning the frozen temperature. On the other hand, the deviation of the $T_{\|} / T_{0}$ profile from zero is too small to convince us that $T_{\|} / T_{0}$ is frozen for $y / \hat{a}_{0}>4$. We come back to this point at the end of the next paragraph. At $y / \hat{a}_{0}=4$ the predicted values of $\hat{T}_{\|}$and $\hat{T}_{\perp}$ are nearly zero; hence a careful estimate of their finiteness from the data in Table I is not required.

Figure 11 shows the results of the data analysis for $T_{\|} / T_{\perp}$ in the case of $u_{p} / a_{0}=6$. Panels (a) and (b) show the time evolution of $T_{\|} / T_{\perp}$ and that of $d \ln \left(T_{\|} / T_{\perp}\right) / d s$ at various values of $y$, respectively. Panel (c) shows the profile of $T_{\|} / T_{\perp}$ in the limit $s \rightarrow \infty$ predicted at $s=2 \times 10^{5}, 1 \times 10^{6}$, and $4 \times 10^{6}$ (solid lines) and the position $y$ in the same limit corresponding to $T_{\|} / T_{\perp}=0.1 n(n=2,3, \ldots, 9)$ predicted at $s=4 \times 10^{6}$ (open circles). As seen from the evolution at $y / \hat{a}_{0}=4.9$ in panel (b), $d \ln \left(T_{\|} / T_{\perp}\right) / d \ln s$ [or $d\left(T_{\|} / T_{\perp}\right) / d s$ ] changes its sign around $y / \hat{a}_{0}=5$; in consequence, the prediction method is not reliable there. Indeed, the predicted values diverge there and thus are not drawn in panel (c). It should be noted that the corresponding part tends to shrink, and an almost converged and smoothly connected profile for the entire $y / \hat{a}_{0}>4$ is obtained. This profile is monotonically decreasing in $y$ and remains finite. The finiteness of $T_{\|} / T_{\perp}$ in the limit $s \rightarrow \infty$ suggests two possibilities: either both $\hat{T}_{\|}$and $\hat{T}_{\perp}$ remain finite or $\hat{T}_{\|}$and $\hat{T}_{\perp}$ vanish with the same rate. Since $\hat{T}_{\perp}$ remains finite as discussed in the previous paragraph, $\hat{T}_{\|}$ can be judged to remain finite as $s \rightarrow \infty$ for $y / \hat{a}_{0}>4$.

Because $T_{\|} / T_{\perp}$ decreases monotonically in $y$ for $y / \hat{a}_{0}>4$ and reaches a smaller value for larger $u_{p} / a_{0}$ [see Figs. 6(c) and 6(d)], $T_{\|} / T_{\perp}$ is likely to vanish in the limit $\left(\hat{u}_{p}, y\right) \rightarrow \infty$. On the other hand, judging from a comparison between $u_{p} / a_{0}=5$ and 6 [18], $T_{\perp} / T_{0}$ is likely to increase in the same limit. Because it is unlikely that $T_{\perp} / T_{0}$ exceeds the value in the free molecular limit, which is unity, $T_{\|} / T_{0}$ should vanish in the limit $\left(\hat{u}_{p}, y\right) \rightarrow \infty$. This limit corresponds to the case that a sonic flow expands into a real vacuum, and thus the frozen perpendicular temperature is suggested in the planar expansion into a vacuum. In order to estimate the degree of the frozen temperature, however, numerical computations for larger values of $u_{p} / a_{0}$ are required. This subject is left unresolved in the present paper.

\section{SUMMARY}

We have numerically investigated the gas behavior induced by rapidly pulling out an adiabatic piston on the basis of the BGK model of the Boltzmann equation, with a particular interest in the long time behavior. We have mainly clarified the following.

(1) If the piston speed is lower than the critical speed $u_{c}(=$ $\left.\frac{\gamma+1}{\gamma-1} a_{0}\right)$, the state of the gas approaches the local Maxwellian corresponding to the expansion wave followed by a uniform state, namely, the prediction of the conventional gas dynamics.

(2) If the piston speed is higher than $u_{c}$, the state of the gas approaches the local Maxwellian corresponding to the expansion wave for $X_{1} / t<u_{c}$, while a strongly nonequilibrium state with a highly low pressure is founded for $X_{1} / t>u_{c}$. In other words, time-asymptotically, there is a critical point $X_{1} / t=u_{c}$ across which the dynamics of the gas behavior changes dramatically. The mass density in the latter region vanishes at infinite time (the formation of vacuum).

(3) In the region $X_{1} / t>u_{c}$, the parallel and perpendicular temperatures are largely different from each other. This anisotropy is not relaxed in the course of time evolution and is stronger for a higher piston speed.

(4) During the time evolution, the parallel and perpendicular temperatures do not monotonically decrease with respect to $X_{1} / t$ from upstream to downstream and can increase for $X_{1} / t>u_{c}$. The ratio of the parallel to the perpendicular temperature remains finite, and thus for any $X_{1} / t$ it does not occur that only one of the component temperatures vanishes.

(5) The temperature decreases slowly in time for $X_{1} / t>$ $u_{c}$, and the parallel and perpendicular temperatures remain finite except in the vicinity of $X_{1} / t=u_{c}$, as far as the piston speed is finite. 


\section{ACKNOWLEDGMENTS}

The present work is partially supported by KAKENHI from the Ministry of Education, Culture, Sports, Science, and Technology in Japan (Grants No. 19560066 and No. 23246034) and by a research grant from the UBE Foundation. A part of the work was done during the stay of the second author at the Centre de Mathématiques Laurent Schwartz, Ecole Polytechnique. He expresses his thanks to Professor François Golse for hospitality, helpful discussions, and drawing his attention to mathematical references.

\section{APPENDIX A: SLOW EVOLUTION AND THE LIMIT FOR LARGE TIME}

As is clear from the numerical results in Sec. V, the solution of Eqs. (6)-(9) approaches a self-similar solution independent of $s$, say $G_{E}(y, \eta)$, with a finite mass density if $\hat{u}_{p}<\hat{u}_{c}$ $\left(u_{p} / a_{0}<4\right)$. Below we set $G(s, y, \eta)=G_{E}(y, \boldsymbol{\eta})+g(s, y, \eta)$ and derive the explicit form of $G_{E}$ by assuming that $|g| \ll$ $\left|G_{E}\right|$ and $\partial_{s} g=O(g / s)$ for large $s$.

Equation (6) can be rewritten by substituting $G(s, y, \eta)=$ $G_{E}(y, \boldsymbol{\eta})+g(s, y, \boldsymbol{\eta})$ as

$$
\begin{gathered}
s \partial_{s} g+\eta_{1}\left(\partial_{y} G_{E}-\partial_{\eta_{1}} G_{E}\right)+\eta_{1}\left(\partial_{y} g-\partial_{\eta_{1}} g\right) \\
=s\left(\hat{\rho}_{E}+\hat{\rho}_{g}\right)\left(\mathcal{G}_{E}-G_{E}+\mathcal{G}_{g}-g\right), \\
\mathcal{G}_{E}=\frac{\hat{\rho}_{E}}{\left(\pi \hat{T}_{E}\right)^{3 / 2}} \exp \left[-\frac{\left(\eta_{1}-v_{E}\right)^{2}+\eta_{2}^{2}+\eta_{3}^{2}}{\hat{T}_{E}}\right] \\
\hat{\rho}_{E}=\int G_{E} d \eta, \\
\hat{\rho}_{E} v_{E}=\hat{\rho}_{E}\left(\hat{u}_{E}-y\right)=\int \eta_{1} G_{E} d \eta, \\
\hat{p}_{E}=\hat{\rho}_{E} \hat{T}_{E}=\frac{2}{3} \int\left[\left(\eta_{1}-v_{E}\right)^{2}+\eta_{2}^{2}+\eta_{3}^{2}\right] G_{E} d \eta, \\
\hat{\rho}_{g}=\int g d \eta, \quad \mathcal{G}_{g}=\mathcal{G}-\mathcal{G}_{E} .
\end{gathered}
$$

Balancing the terms in Eq. (A1a) yields

$$
\begin{aligned}
s \hat{\rho}_{E}\left(\mathcal{G}_{E}-G_{E}\right) & =0, \\
\eta_{1}\left(\partial_{y} G_{E}-\partial_{\eta_{1}} G_{E}\right) & =s \hat{\rho}_{g}\left(\mathcal{G}_{E}-G_{E}\right)+s \hat{\rho}_{E}\left(\mathcal{G}_{g}-g\right), \\
s \partial_{s} g+\eta_{1}\left(\partial_{y} g-\partial_{\eta_{1}} g\right) & =s \hat{\rho}_{g}\left(\mathcal{G}_{g}-g\right) .
\end{aligned}
$$

From Eq. (A2a), $G_{E}=\mathcal{G}_{E}$, namely, $G_{E}$ is a local Maxwellian; accordingly Eq. (A2b) is reduced to

$$
\eta_{1}\left(\partial_{y} \mathcal{G}_{E}-\partial_{\eta_{1}} \mathcal{G}_{E}\right)=s \hat{\rho}_{E}\left(\mathcal{G}_{g}-g\right) .
$$

The integrations of this equation multiplied by $1, \eta_{1},|\eta|^{2}$ over the whole $\eta$ yield

$$
\begin{aligned}
v_{E} \partial_{y} \hat{\rho}_{E}+\hat{\rho}_{E}\left(1+\partial_{y} v_{E}\right) & =0, \\
\frac{1}{2} \partial_{y} \hat{p}_{E}+\hat{\rho}_{E} v_{E}\left(1+\partial_{y} v_{E}\right) & =0, \\
\frac{5}{2} v_{E} \partial_{y} \hat{p}_{E}+\left(\frac{5}{2} \hat{p}_{E}+2 \hat{\rho}_{E} v_{E}^{2}\right)\left(1+\partial_{y} v_{E}\right) & =0 .
\end{aligned}
$$

From Eqs. (A3b) and (A3c), we eventually obtain

$$
\hat{p}_{E}=\frac{6}{5} \hat{\rho}_{E} v_{E}^{2} \quad \text { or } \quad v_{E}=-y+\text { const. }
$$

When $\hat{p}_{E}=\frac{6}{5} \hat{\rho}_{E} v_{E}^{2}$, Eq. (A3b) reduces, with the aid of Eq. (A3a), to

$$
\hat{\rho}_{E} v_{E}\left(1+4 \partial_{y} v_{E}\right)=0 .
$$

Thanks to Eq. (A3a), $\hat{\rho}_{E} v_{E}=0$ implies $\hat{\rho}_{E}=0$. Hence we exclude this case and conclude $1+4 \partial_{y} v_{E}=0$, namely,

$$
v_{E}=-\frac{1}{4} y+c_{0} \quad\left(\text { or } \hat{u}_{E}=\frac{3}{4} y+c_{0}\right) .
$$

By the substitution into Eq. (A3), $\hat{\rho}_{E}, \hat{p}_{E}$, and $\hat{T}_{E}$ are easily obtained as

$$
\begin{gathered}
\hat{\rho}_{E}=c_{1} c_{0}^{3}\left(1-\frac{1}{4} \frac{y}{c_{0}}\right)^{3}, \hat{p}_{E}=\frac{6}{5} c_{1} c_{0}^{5}\left(1-\frac{1}{4} \frac{y}{c_{0}}\right)^{5}, \\
\hat{T}_{E}=\frac{6}{5} c_{0}^{2}\left(1-\frac{1}{4} \frac{y}{c_{0}}\right)^{2} .
\end{gathered}
$$

Finally, by assuming the sonic condition at $y=0$, the constants $c_{0}$ and $c_{1}$ are determined consistently as $c_{0}=\hat{a}_{0}=\sqrt{5 / 6}$ and $c_{1}=c_{0}^{-3}$. Therefore Eq. (A5) is the solution of the expansion wave explained in Sec. IV A. In the meantime, when $v_{E}=$ $-y+$ const, all of $\hat{\rho}_{E}, \hat{p}_{E}$, and $\hat{T}_{E}$ become constants. This case corresponds to the uniform state behind the expansion tail. The constants are determined so that the uniform state is connected to the expansion tail at $y=\frac{4}{3}\left(\hat{u}_{p}-\hat{a}_{0}\right)$ where $\hat{u}_{E}=\hat{u}_{p}$. This completes the construction of $G_{E}$, and $G_{E}$ is shown to be the local Maxwellian corresponding to the expansion wave and its downstream uniform state obtained by the conventional gas dynamics.

\section{APPENDIX B: NUMERICAL SCHEME AND DATA OF COMPUTATION}

The initial- and boundary-value problem in Sec. III can be reduced to the following set of equations

$$
\begin{aligned}
& s \partial_{s} \Phi+\eta_{1}\left(\partial_{y} \Phi-\partial_{\eta_{1}} \Phi\right) \\
& =s \hat{\rho}(\Psi-\Phi),\left(0<y<\hat{u}_{p}\right), \\
& \Psi=\left[\begin{array}{c}
1 \\
\hat{T}
\end{array}\right] \frac{\hat{\rho}}{(\pi \hat{T})^{1 / 2}} \exp \left(-\frac{\left(\eta_{1}-v\right)^{2}}{\hat{T}}\right), \\
& \hat{\rho}=\int_{-\infty}^{\infty} g d \eta_{1}, \quad \hat{\rho} v=\int_{-\infty}^{\infty} \eta_{1} g d \eta_{1}, \\
& \hat{p}=\hat{\rho} \hat{T}=\frac{2}{3} \int_{-\infty}^{\infty}\left[\left(\eta_{1}-v\right)^{2} g+h\right] d \eta_{1},
\end{aligned}
$$

boundary conditions

$$
\begin{gathered}
\Phi=\Phi_{A}, \quad\left(y=0, \eta_{1}>0\right), \\
\Phi\left(\eta_{1}\right)=\Phi\left(-\eta_{1}\right), \quad\left(y=\hat{u}_{p}, \eta_{1}<0\right),
\end{gathered}
$$




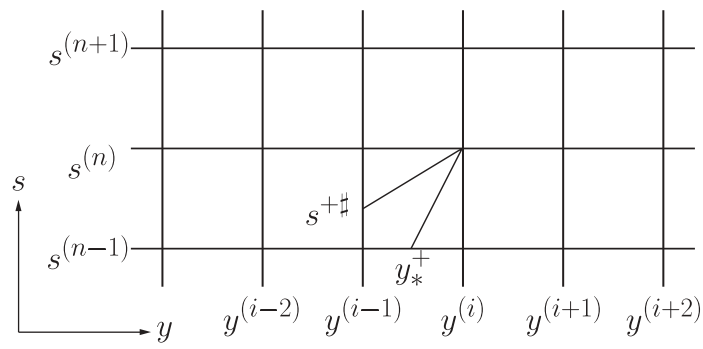

(a)

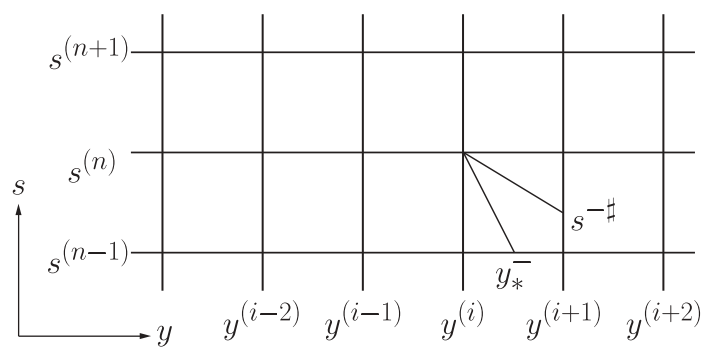

(b)

FIG. 12. Sketch of the transport process. (a) $\eta_{1}^{(j)}>0$ and (b) $\eta_{1}^{(j)}<0$. In panel (a), the characteristic curve passing through $\left(s, y, \eta_{1}\right)=$ $\left(s^{(n)}, y^{(i)}, \eta_{1}^{(j)}\right)$ intersects the line $s=s^{(n-1)}$ or $y=y^{(i-1)}$, according to $\eta_{1}^{(j)} \lessgtr \eta_{c}^{+}=\left(y^{(i)}-y^{(i-1)}\right) s^{(n-1)} / \delta s^{(n)}$. In panel (b), the characteristic curve passing through $\left(s, y, \eta_{1}\right)=\left(s^{(n)}, y^{(i)}, \eta_{1}^{(j)}\right)$ intersects the line $s=s^{(n-1)}$ or $y=y^{(i+1)}$, according to $\left|\eta_{1}^{(j)}\right| \lessgtr \eta_{c}^{-}=\left(y^{(i+1)}-y^{(i)}\right) s^{(n-1)} / \delta s^{(n)}$.

and initial conditions

$$
\Phi= \begin{cases}\Phi_{A}, & \left(\eta_{1}>0\right), \\ \Phi_{B}, & \left(\eta_{1}<0\right),\end{cases}
$$

for the marginal velocity distribution functions. Here,

$$
\begin{aligned}
\Phi & =\left[\begin{array}{l}
g \\
h
\end{array}\right], \Phi_{A}=\left[\begin{array}{l}
1 \\
1
\end{array}\right] \frac{1}{\pi^{1 / 2}} \exp \left(-\left(\eta_{1}-v_{0}\right)^{2}\right), \\
\Phi_{B} & =\Phi_{A}\left(\eta_{1}-2\left(\eta_{1}-v_{p}\right)\right) .
\end{aligned}
$$

We solve the problem, Eqs. (B1)-(B3), numerically.

In numerical computations, the $\eta_{1}$ space is limited to a finite interval $\left|\eta_{1}\right| \leqslant \eta_{D}$, where $\eta_{D}$ is chosen to be sufficiently large so as for $\Phi$ to be negligibly small around $\left|\eta_{1}\right| \sim$ $\eta_{D}$. Grid points are arranged in the $y$ and $\eta_{1}$ spaces as $0=y^{(0)}<y^{(1)}<\cdots<y^{(N)}=\hat{u}_{p}$ and $\eta_{1}^{(-2 M)}<\eta_{1}^{(-2 M+1)}<$ $\cdots<\eta_{1}^{(0)}<\eta_{1}^{(1)}<\cdots<\eta_{1}^{(2 M)}$. Here $\eta_{1}^{(0)}=0$ and $\eta_{1}^{(2 M)}=$ $-\eta_{1}^{(-2 M)}=\eta_{D}$. In what follows, the part $\eta_{1} \gtrless 0$ of $\Phi$ and $\Psi$ will be denoted by $\Phi^{ \pm}$and $\Psi^{ \pm}$, and the value of quantities on a grid point at time $s=s^{(n)}(n=0,1, \ldots)$ will be denoted by $\Phi_{(i, j)}^{ \pm(n)}=\Phi\left(s^{(n)}, y^{(i)}, \eta_{1}^{( \pm j)}\right), \quad \Psi_{(i, j)}^{ \pm(n)}=$ $\Psi\left(s^{(n)}, y^{(i)}, \eta_{1}^{( \pm j)}\right)$, and $h_{(i)}^{(n)}=h\left(s^{(n)}, y^{(i)}\right)$, where $h=\hat{\rho}, v$, etc. $\lim _{\eta_{1} \rightarrow \pm 0} \Phi^{ \pm}\left(s^{(n)}, y^{(i)}, \eta_{1}\right)$ and $\lim _{\eta_{1} \rightarrow \pm 0} \Psi^{ \pm}\left(s^{(n)}, y^{(i)}, \eta_{1}\right)$ will be denoted by $\Phi_{(i, 0)}^{ \pm(n)}$ and $\Psi_{(i, 0)}^{ \pm(n)}$, because there is a discontinuity at $\eta_{1}=0$. We numerically construct $\Phi_{(i, j)}^{ \pm(n)}(n=1,2, \ldots)$, starting from $\Phi_{(i, j)}^{ \pm(0)}$, by the method described below.

\section{Splitting method}

The scheme is designed on the basis of the splitting method. To obtain $\Phi$ at $s=s^{(n)}$, Eq. (B1a) is integrated, after dropping its right-hand side, along the characteristics over the interval $\delta s^{(n)}=s^{(n)}-s^{(n-1)}$ with the data at the previous step $s=$ $s^{(n-1)}$ being the initial condition (the transport process). Then, Eq. (B1a) is integrated, after dropping the second term of its left-hand side, over the same time interval with the solution of the transport process being the initial condition (the collision process). The details of each process are given below, where $\Phi_{\left(i^{\prime}, j^{\prime}\right)}^{ \pm(n-1)}\left(i^{\prime}=0, \ldots, N ; j^{\prime}=0, \ldots, 2 M\right)$ and $\Phi_{\left(i \mp 1, j^{\prime}\right)}^{ \pm(n)}\left(j^{\prime}=\right.$ $0, \ldots, 2 M)$ are supposed to be known.

(a) Transport process. Since $\Phi^{ \pm}$does not change along the characteristics of Eq. (B1a), the value of $\Phi^{ \pm}$on the grid point $\left(s, y, \eta_{1}\right)=\left(s^{(n)}, y^{(i)}, \eta_{1}^{( \pm j)}\right)$, which we denote by $\Phi_{(i, j)}^{ \pm *}$, can be given by the value at the upstream position (Fig. 12), i.e.,

$$
\Phi_{(i, j)}^{ \pm *}= \begin{cases}\Phi^{ \pm}\left(s^{(n-1)}, y_{*}^{ \pm}, \eta_{1}^{ \pm *}\right), & \text { for }\left|\eta_{1}^{( \pm j)}\right| \leqslant \eta_{c}^{ \pm}, \\ \Phi^{ \pm}\left(s^{ \pm \sharp}, y^{(i \mp 1)}, \eta_{1}^{ \pm \sharp}\right), & \text { for }\left|\eta_{1}^{( \pm j)}\right|>\eta_{c}^{ \pm} .\end{cases}
$$

Here, $\eta_{c}^{ \pm}=\left|y^{(i)}-y^{(i \mp 1)}\right| s^{(n-1)} / \delta s^{(n)}, y_{*}^{ \pm}=y^{(i)}-\eta_{1}^{( \pm j)} \delta s^{(n)} \mid$ $s^{(n-1)}, \eta_{1}^{ \pm *}=\eta_{1}^{( \pm j)} s^{(n)} / s^{(n-1)}, \eta_{1}^{ \pm \#}=y^{(i)}-y^{(i \mp 1)}+\eta_{1}^{( \pm j)}$, and $s^{ \pm \sharp}=s^{(n)} \eta_{1}^{( \pm j)} / \eta_{1}^{ \pm \sharp}$. In the case of $\left|\eta_{1}^{( \pm j)}\right| \leqslant \eta_{c}^{ \pm}$, since $\Phi_{\left(i^{\prime}, j^{\prime}\right)}^{ \pm(n-1)}$ $\left(i^{\prime}=0, \ldots, N ; j^{\prime}=0, \ldots, 2 M\right)$ is known, $\Phi^{ \pm}\left(s^{(n-1)}, y_{*}^{ \pm}, \eta_{1}^{ \pm *}\right)$ can be obtained by interpolations with respect to $y$ and $\eta_{1}$. In the case of $\left|\eta_{1}^{( \pm j)}\right|>\eta_{c}^{ \pm}, \Phi^{ \pm}\left(s^{(n-1)}, y^{(i \mp 1)}, \eta_{1}^{ \pm \sharp}\right)$ and $\Phi^{ \pm}\left(s^{(n)}, y^{(i \mp 1)}, \eta_{1}^{ \pm \sharp}\right)$ are first computed by interpolations with respect to $\eta_{1}$, respectively, from $\Phi_{\left(i \mp 1, j^{\prime}\right)}^{ \pm(n-1)}$ and $\Phi_{\left(i \mp 1, j^{\prime}\right)}^{ \pm(n)}\left(j^{\prime}=\right.$ $0, \ldots, 2 M)$. Then $\Phi^{ \pm}\left(s^{ \pm \sharp}, y^{(i \mp 1)}, \eta_{1}^{ \pm \sharp}\right)$ is obtained by the linear interpolation:

$$
\begin{aligned}
\Phi^{ \pm}\left(s^{ \pm \sharp}, y^{(i \mp 1)}, \eta_{1}^{ \pm \sharp}\right)= & \frac{\delta s^{ \pm \sharp}}{\delta s^{(n)}} \Phi^{ \pm}\left(s^{(n-1)}, y^{(i \mp 1)}, \eta_{1}^{ \pm \sharp}\right) \\
& +\left(1-\frac{\delta s^{ \pm \sharp}}{\delta s^{(n)}}\right) \Phi^{ \pm}\left(s^{(n)}, y^{(i \mp 1)}, \eta_{1}^{ \pm \sharp}\right),
\end{aligned}
$$

where $\delta s^{ \pm \sharp}=s^{(n)}-s^{ \pm \sharp}$.

For $(i, j)$ such that $\left|\eta_{1}^{ \pm *}\right|>\eta_{D}$ or $\left|\eta_{1}^{ \pm \sharp}\right|>\eta_{D}, \Phi_{(i, j)}^{ \pm *}=0$ is applied in place of Eq. (B5).

(b) Collision process. $\Phi_{(i, j)}^{ \pm(n)}$ is computed by

$$
\Phi_{(i, j)}^{ \pm(n)}=\Phi_{(i, j)}^{ \pm *}+\delta s^{ \pm *} \hat{\rho}_{(i)}^{(n-1)}\left[\Psi_{(i, j)}^{ \pm(n-1)}-\Phi_{(i, j)}^{ \pm(n-1)}\right],
$$

where

$$
\delta s^{ \pm *}= \begin{cases}\delta s^{(n)}, & \text { for }\left|\eta_{1}^{( \pm j)}\right| \leqslant \eta_{c}^{ \pm} \\ \delta s^{ \pm \sharp}, & \text { for }\left|\eta_{1}^{( \pm j)}\right|>\eta_{c}^{ \pm} .\end{cases}
$$

\section{Procedure of computation}

The procedure of computation is as follows.

(i) Prepare $\Phi_{(i, j)}^{ \pm(0)}(i=0, \ldots, N ; j=0, \ldots, 2 M)$ by using initial condition (B3).

(ii) Compute $h_{(i)}^{(0)}(i=0, \ldots, N)$ from $\Phi_{(i, j)}^{ \pm(0)}$ by using Eqs. (B1c) and (B1d).

(iii) Compute $\Phi_{(0, j)}^{+(1)}(j=0, \ldots, 2 M)$ by using boundary condition (B2a). 
TABLE II. Variation in the macroscopic variables at several $y$ and $s$ when the number of grid points in $y($ i.e., $N)$ is doubled $\left(u_{p} / a_{0}=6\right)$. The value in the square brackets represents the actual value of $\hat{\rho}, v, \hat{T}_{\|}$, or $\hat{T}_{\perp}$.

\begin{tabular}{|c|c|c|c|c|c|c|c|c|c|c|c|}
\hline \multirow[b]{2}{*}{$s \backslash$} & \multirow[b]{2}{*}{$y / \hat{a}_{0}$} & \multicolumn{10}{|c|}{$\hat{\rho}$} \\
\hline & & & .0 & & & & 0 & & & 6 & \\
\hline $1 \times 10^{2}$ & & $2.1(-5)^{\mathrm{a}}$ & {$[4.3(-1)]$} & $3.6(-7)$ & {$[1.4(-2)]$} & $9.0(-8)$ & {$[2.9(-4)]$} & $3.1(-10)$ & {$[6.8(-7)]$} & $8.5(-13)$ & {$[7.3(-10)]$} \\
\hline $1 \times 10^{4}$ & & $6.2(-7)$ & {$[4.2(-1)]$} & $3.5(-7)$ & {$[1.5(-2)]$} & $2.9(-7)$ & {$[1.7(-4)]$} & $1.1(-8)$ & {$[1.4(-6)]$} & $1.1(-11)$ & {$[1.0(-9)]$} \\
\hline $1 \times 10^{6}$ & & $9.2(-9)$ & {$[4.2(-1)]$} & $1.5(-10)$ & {$[1.6(-2)]$} & $9.7(-9)$ & {$[7.7(-6)]$} & $5.8(-10)$ & {$[9.1(-8)]$} & $5.7(-13)$ & {$[4.0(-11)]$} \\
\hline
\end{tabular}

\begin{tabular}{|c|c|c|c|c|c|c|c|c|c|c|c|}
\hline \multirow[b]{2}{*}{$s \backslash$} & \multirow[b]{2}{*}{$y / \hat{a}_{0}$} & \multicolumn{10}{|c|}{$v$} \\
\hline & & & 0 & & & & 1.0 & & & & \\
\hline$\overline{1 \times 10^{2}}$ & & $2.6(-5)$ & {$[6.7(-1)]$} & $3.2(-4)$ & {$[3.3(-1)]$} & $3.5(-4)$ & {$[2.6(-1)]$} & $2.4(-4)$ & {$[2.1(-1)]$} & - & [0] \\
\hline $1 \times 10^{4}$ & & $5.6(-7)$ & {$[6.8(-1)]$} & $5.1(-6)$ & {$[2.3(-1)]$} & $1.6(-6)$ & {$[9.6(-2)]$} & $1.1(-4)$ & {$[8.6(-2)]$} & - & [0] \\
\hline $1 \times 10^{6}$ & & $1.3(-8)$ & {$[6.8(-1)]$} & $1.8(-8)$ & {$[2.3(-1)]$} & $4.2(-7)$ & {$[2.1(-2)]$} & $1.2(-5)$ & {$[3.1(-2)]$} & - & [0] \\
\hline
\end{tabular}

\begin{tabular}{|c|c|c|c|c|c|c|c|c|c|c|c|}
\hline \multirow{2}{*}{$\begin{array}{r}s \backslash \\
\times 10^{2}\end{array}$} & $y / \hat{a}_{0}$ & & .0 & & & & t.0 & & & & \\
\hline & & $4.1(-6)$ & {$[5.7(-1)]$} & $2.0(-4)$ & {$[1.5(-1)]$} & $1.5(-4)$ & {$[7.4(-2)]$} & $1.2(-4)$ & {$[4.8(-2)]$} & $1.8(-4)$ & {$[9.5(-2)]$} \\
\hline $1 \times 10^{4}$ & & $2.5(-7)$ & {$[5.6(-1)]$} & $1.7(-6)$ & {$[6.5(-2)]$} & $2.7(-5)$ & {$[3.2(-2)]$} & $2.6(-5)$ & {$[1.5(-2)]$} & $4.4(-5)$ & {$[1.2(-2)]$} \\
\hline $1 \times 10^{6}$ & & $6.1(-9)$ & {$[5.6(-1)]$} & $7.5(-8)$ & {$[6.3(-2)]$} & $1.6(-7)$ & {$[3.5(-3)]$} & $1.5(-5)$ & {$[6.5(-3)]$} & $8.0(-6)$ & {$[1.8(-3)]$} \\
\hline
\end{tabular}

\begin{tabular}{|c|c|c|c|c|c|c|c|c|c|c|c|}
\hline \multirow{2}{*}{$\begin{array}{r}s \backslash \\
1 \times 10^{2}\end{array}$} & \multirow[t]{2}{*}{$y / \hat{a}_{0}$} & \multicolumn{2}{|c|}{1.0} & \multicolumn{2}{|c|}{3.0} & \multicolumn{2}{|c|}{4.0} & \multicolumn{2}{|c|}{5.0} & \multicolumn{2}{|c|}{6.0} \\
\hline & & $6.7(-6)$ & {$[5.9(-1)]$} & $4.7(-4)$ & {$[3.8(-1)]$} & $4.2(-4)$ & {$[5.7(-1)]$} & $3.5(-4)$ & {$[6.4(-1)]$} & $2.3(-4)$ & {$[6.7(-1)]$} \\
\hline $1 \times 10^{4}$ & & $2.5(-7)$ & {$[5.6(-1)]$} & $1.7(-6)$ & {$[6.6(-2)]$} & $3.0(-5)$ & {$[8.3(-2)]$} & $3.5(-5)$ & {$[2.6(-1)]$} & $4.5(-5)$ & {$[3.6(-1)]$} \\
\hline $1 \times 10^{6}$ & & $6.1(-9)$ & {$[5.6(-1)]$} & $7.5(-8)$ & {$[6.3(-2)]$} & $1.1(-6)$ & {$[4.4(-3)]$} & $4.1(-6)$ & {$[1.3(-1)]$} & $1.6(-4)$ & {$[2.4(-1)]$} \\
\hline
\end{tabular}

${ }^{\mathrm{a}} \operatorname{Read}$ as $2.1 \times 10^{-5}$.

TABLE III. Variation in the macroscopic variables at several $y$ and $s$ when the twice larger time interval than in the standard case is used $\left(u_{p} / a_{0}=6\right)$. The value in the square brackets represents the actual value of $\hat{\rho}, v, \hat{T}_{\|}$, or $\hat{T}_{\perp}$.

\begin{tabular}{|c|c|c|c|c|c|c|c|c|c|c|c|}
\hline \multirow{3}{*}{$\frac{s \backslash}{1 \times 10^{2}}$} & \multirow{3}{*}{$y / \hat{a}_{0}$} & \multicolumn{10}{|c|}{$\hat{\rho}$} \\
\hline & & \multicolumn{2}{|c|}{1.0} & \multicolumn{2}{|c|}{3.0} & \multicolumn{2}{|c|}{4.0} & \multicolumn{2}{|c|}{5.0} & \multicolumn{2}{|c|}{6.0} \\
\hline & & $5.5(-5)^{\mathrm{a}}$ & {$[4.3(-1)]$} & $1.7(-6)$ & {$[1.4(-2)]$} & $1.1(-7)$ & {$[2.9(-4)]$} & $1.3(-9)$ & {$[6.8(-7)]$} & $2.7(-12)$ & {$[7.3(-10)]$} \\
\hline $1 \times 10^{4}$ & & $1.3(-6)$ & {$[4.2(-1)]$} & $4.7(-6)$ & {$[1.5(-2)]$} & $6.6(-7)$ & {$[1.7(-4)]$} & $3.7(-9)$ & {$[1.4(-6)]$} & $5.1(-12)$ & {$[1.0(-9)]$} \\
\hline $1 \times 10^{6}$ & & $2.4(-8)$ & {$[4.2(-1)]$} & $1.4(-7)$ & {$[1.6(-2)]$} & $6.5(-8)$ & {$[7.7(-6)]$} & $4.2(-10)$ & {$[9.1(-8)]$} & $2.4(-13)$ & {$[4.0(-11)]$} \\
\hline \multirow[t]{2}{*}{$4 \times 10^{6}$} & & $1.6(-9)$ & {$[4.2(-1)]$} & $3.7(-8)$ & {$[1.6(-2)]$} & $2.8(-8)$ & {$[3.0(-6)]$} & $1.3(-10)$ & {$[2.9(-8)]$} & $7.6(-14)$ & {$[1.2(-11)]$} \\
\hline & & \multicolumn{10}{|c|}{$v$} \\
\hline$s \backslash$ & $y / \hat{a}_{0}$ & \multicolumn{2}{|c|}{1.0} & \multicolumn{2}{|c|}{3.0} & \multicolumn{2}{|c|}{4.0} & \multicolumn{2}{|c|}{5.0} & \multicolumn{2}{|c|}{6.0} \\
\hline$\overline{1 \times 10^{2}}$ & & $2.2(-4)$ & {$[6.7(-1)]$} & $9.2(-5)$ & {$[3.3(-1)]$} & $9.9(-5)$ & {$[2.6(-1)]$} & $9.7(-5)$ & {$[2.1(-1)]$} & - & [0] \\
\hline $1 \times 10^{4}$ & & $6.4(-6)$ & {$[6.8(-1)]$} & $8.7(-5)$ & {$[2.3(-1)]$} & $1.6(-4)$ & {$[9.6(-2)]$} & $1.1(-4)$ & {$[8.6(-2)]$} & - & {$[0]$} \\
\hline $1 \times 10^{6}$ & & $1.2(-7)$ & {$[6.8(-1)]$} & $2.1(-6)$ & {$[2.3(-1)]$} & $1.9(-5)$ & {$[2.1(-2)]$} & $2.4(-5)$ & {$[3.1(-2)]$} & - & {$[0]$} \\
\hline \multirow[t]{2}{*}{$4 \times 10^{6}$} & & $4.5(-8)$ & {$[6.8(-1)]$} & $7.0(-7)$ & {$[2.3(-1)]$} & $1.5(-5)$ & {$[1.4(-2)]$} & $8.9(-6)$ & {$[2.4(-2)]$} & - & {$[0]$} \\
\hline & & \multicolumn{10}{|c|}{$\hat{T}_{\|}$} \\
\hline$s \backslash$ & $y / \hat{a}_{0}$ & \multicolumn{2}{|c|}{1.0} & \multicolumn{2}{|c|}{3.0} & \multicolumn{2}{|c|}{4.0} & \multicolumn{2}{|c|}{5.0} & \multicolumn{2}{|c|}{6.0} \\
\hline$\overline{1 \times 10^{2}}$ & & $5.4(-4)$ & {$[5.7(-1)]$} & $1.6(-4)$ & {$[1.5(-1)]$} & $1.5(-5)$ & {$[7.4(-2)]$} & $1.0(-6)$ & {$[4.8(-2)]$} & $2.5(-4)$ & {$[9.5(-2)]$} \\
\hline $1 \times 10^{4}$ & & $8.7(-6)$ & {$[5.6(-1)]$} & $7.0(-5)$ & {$[6.5(-2)]$} & $2.1(-5)$ & {$[3.2(-2)]$} & $2.1(-5)$ & {$[1.5(-2)]$} & $1.8(-5)$ & {$[1.2(-2)]$} \\
\hline $1 \times 10^{6}$ & & $1.7(-7)$ & {$[5.6(-1)]$} & $6.6(-7)$ & {$[6.3(-2)]$} & $1.3(-5)$ & {$[3.5(-3)]$} & $6.4(-6)$ & {$[6.5(-3)]$} & $2.2(-6)$ & {$[1.8(-3)]$} \\
\hline $4 \times 10^{6}$ & & $7.7(-8)$ & {$[5.6(-1)]$} & $2.0(-7)$ & {$[6.3(-2)]$} & $6.6(-6)$ & {$[1.5(-3)]$} & $5.0(-6)$ & {$[5.5(-3)]$} & $1.3(-6)$ & {$[1.2(-3)]$} \\
\hline & & & & & & & & & & & \\
\hline
\end{tabular}

\begin{tabular}{|c|c|c|c|c|c|c|c|c|c|c|c|}
\hline$s \backslash$ & $y / \hat{a}_{0}$ & \multicolumn{2}{|c|}{1.0} & \multicolumn{2}{|c|}{3.0} & \multicolumn{2}{|c|}{4.0} & \multicolumn{2}{|c|}{5.0} & \multicolumn{2}{|c|}{6.0} \\
\hline$\overline{1 \times 10^{2}}$ & & $5.5(-4)$ & {$[5.9(-1)]$} & $6.3(-5)$ & {$[3.8(-1)]$} & $4.8(-4)$ & {$[5.7(-1)]$} & $5.1(-4)$ & {$[6.4(-1)]$} & $4.2(-4)$ & {$[6.7(-1)]$} \\
\hline $1 \times 10^{4}$ & & $8.7(-6)$ & {$[5.6(-1)]$} & $7.1(-5)$ & {$[6.6(-2)]$} & $1.7(-4)$ & {$[8.3(-2)]$} & $4.0(-5)$ & {$[2.6(-1)]$} & $2.6(-4)$ & {$[3.6(-1)]$} \\
\hline $1 \times 10^{6}$ & & $1.7(-7)$ & {$[5.6(-1)]$} & $6.6(-7)$ & {$[6.3(-2)]$} & $2.5(-5)$ & {$[4.4(-3)]$} & $1.4(-4)$ & {$[1.3(-1)]$} & $4.8(-5)$ & {$[2.4(-1)]$} \\
\hline $4 \times 10^{6}$ & & $7.7(-8)$ & {$[5.6(-1)]$} & $2.0(-7)$ & {$[6.3(-2)]$} & $1.0(-5)$ & {$[1.8(-3)]$} & $1.5(-4)$ & {$[1.1(-1)]$} & $3.7(-5)$ & {$[2.2(-1)]$} \\
\hline
\end{tabular}

${ }^{\mathrm{a}}$ Read as $5.5 \times 10^{-5}$. 
(iv) Compute $\Phi_{(i, j)}^{+(1)}(j=0, \ldots, 2 M)$ by the method described in Sec. B1 in ascending order from $i=1$ to $N$.

(v) Compute $\Phi_{(N, j)}^{-(1)}(j=0, \ldots, 2 M)$ by using boundary condition (B2b).

(vi) Compute $\Phi_{(i, j)}^{-(1)}(j=0, \ldots, 2 M)$ by the method described in Sec. B1 in descending order from $i=N-1$ to 0 .

(vii) Repeat steps (ii)-(vi) by adding unity to superscript numbers in parentheses.

\section{Accuracy of numerical data}

The parameters used in the actual computations are summarized as follows. We used a uniform grid system for the $y$ space and set $N=600$ for the case $u_{p} / a_{0}=3$ and $N=\left(u_{p} / a_{0}\right) \times 100$ otherwise. For the discretization of the $\eta_{1}$ space, we set $2 M=700$ irrespective of $u_{p} / a_{0}$ and used nonuniform grids in the interval $\left|\eta_{1}\right| \leqslant \eta_{D}$. Here, the distribution of $\left\{\eta_{1}^{(j)}\right\}$ is symmetric about $\eta_{1}^{(0)}=0$, i.e., $\eta_{1}^{(-j)}=$ $-\eta_{1}^{(j)}(j=0, \ldots, 2 M)$, and $\eta_{D}$ is about 9.23. The grid points $\left\{\eta_{1}^{(j)}\right\}$ are concentrated near $\eta_{1}^{(0)}=0$; the minimum (or the maximum) grid interval is about $1.58 \times 10^{-5}$ (or 0.04), taking place between $\eta_{1}^{(0)}$ and $\eta_{1}^{(1)}$ [or between $\eta_{1}^{(2 M-1)}$ and $\eta_{1}^{(2 M)}$ ]. For $s$, we set $s^{(n+1)}=s^{(n)}+\Delta s_{(n)}(n=0,1, \ldots)$, where $s^{(0)}=0$ and $\Delta s_{(n)}$ is a variable time step suitably chosen depending on various stages in the time evolution. We increased $\Delta s_{(n)}$ from 0.1 to 0.4 stepwise in the course of numerical computation.

The accuracy of the numerical data is assessed by looking at the variation in the macroscopic variables when the number of the grid points ( $N$ or $2 M$ ) is increased (or decreased) or when the time interval $\Delta s_{(n)}$ is changed. Here, we only show the results for the dependencies on $N$ and $\Delta s_{(n)}$ in the case of $u_{p} / a_{0}=6$. The variation when the number of the $\eta_{1}$ grid points (i.e., $2 M$ ) is changed was much smaller than the corresponding variation resulting from the change of $N$ or $\Delta s_{(n)}$. Table II shows the variation in the macroscopic variables $\hat{\rho}, v, \hat{T}_{\|}$, and $\hat{T}_{\perp}$ at certain locations $y$ at several instances when $N$ is doubled (i.e., the grid spacing is halved) from the value in the standard system mentioned above. Similarly, Table III shows the same variation at certain locations $y$ at several instances when the time interval is made twice larger than in the standard case mentioned above. In the tables, the actual values of the macroscopic variables at the corresponding locations and instances are also shown for reference. The level of accuracy is similar for other cases with different $u_{p} / a_{0}$.
[1] H. W. Liepmann and A. Roshko, Elements of Gasdynamics (Dover, New York, 2001).

[2] S. Takata, Bull. Inst. Math. Acad. Sin. (N. S.) 3, 115 (2008).

[3] B. B. Hamel and D. R. Willis, Phys. Fluids 9, 829 (1966).

[4] R. H. Edwards and H. K. Cheng, AIAA J. 4, 558 (1966).

[5] Y. Sone and H. Sugimoto, Phys. Fluids 7, 2072 (1995).

[6] Y. Sone and H. Sugimoto, Phys. Fluids A 5, 1491 (1993).

[7] R. Illner and M. Shinbrot, Commun. Math. Phys. 94, 217 (1984).

[8] G. Toscani, Arch. Ration. Mech. Anal. 95, 37 (1986).

[9] S.-Y. Ha, Bull. Inst. Math. Acad. Sin. (N. S.) 2, 221 (2007).

[10] T. Yang, J. Comput. Appl. Math. 190, 211 (2006).

[11] B. Einfeldt, C. D. Munz, P. L. Roe, and B. Sjögreen, J. Comput. Phys. 92, 273 (1991).
[12] C. D. Munz, Math. Methods Appl. Sci. 17, 597 (1994).

[13] A. L. Velikovich, S. T. Zalesak, N. Metzler, and J. G. Wouchuk, Phys. Rev. E 72, 046306 (2005).

[14] D. M. Moody, J. Fluid Mech. 214, 455 (1990).

[15] P. L. Bhatnagar, E. P. Gross, and M. Krook, Phys. Rev. 94, 511 (1954).

[16] P. Welander, Ark. Fys. 7, 507 (1954).

[17] C. M. Cuesta and C. Schmeiser, Monatsh Math. 160, 361 (2010).

[18] Although the results for $u_{p} / a_{0}=5$ corresponding to Fig. 10 are not presented in the paper, $T_{\perp} / T_{0}$ in the limit $s \rightarrow \infty$ predicted at $s=4 \times 10^{6}$ monotonically increases in $y$ from nearly zero to about 0.03 in the region $4 \leqslant y / \hat{a}_{0} \leqslant 5$. 